\title{
PERFORATION CONDITIONS AND ALMOST ALGEBRAIC ORDER IN CUNTZ SEMIGROUPS
}

\author{
RAMON ANTOINE, FRANCESC PERERA, AND HENNING PETKZA
}

\begin{abstract}
For a $\mathrm{C}^{*}$-algebra $A$, it is an important problem to determine the Cuntz semigroup $\mathrm{Cu}(A \otimes \mathcal{Z})$ in terms of $\mathrm{Cu}(A)$. We approach this problem from the point of view of semigroup tensor products in the category of abstract Cuntz semigroups, by analysing the passage of significant properties from $\mathrm{Cu}(A)$ to $\mathrm{Cu}(A) \otimes_{\mathrm{Cu}} \mathrm{Cu}(\mathcal{Z})$. We describe the effect of the natural map $\mathrm{Cu}(A) \rightarrow \mathrm{Cu}(A) \otimes_{\mathrm{Cu}} \mathrm{Cu}(\mathcal{Z})$ in the order of $\mathrm{Cu}(A)$, and show that, if $A$ has real rank zero and no elementary subquotients, $\mathrm{Cu}(A) \otimes_{\mathrm{Cu}} \mathrm{Cu}(\mathcal{Z})$ enjoys the corresponding property of having a dense set of (equivalence classes of) projections. In the simple, nonelementary, real rank zero and stable rank one situation, our investigations lead us to identify almost unperforation for projections with the fact that tensoring with $\mathcal{Z}$ is inert at the level of the Cuntz semigroup.
\end{abstract}

\section{INTRODUCTION}

Conditions of perforation in the Cuntz semigroup of a $\mathrm{C}^{*}$-algebra and tensorial absorption of the $\mathrm{C}^{*}$-algebra $\mathcal{Z}$, constructed by Jiang and $\mathrm{Su}$ [19], play an essential part in the classification theory of $\mathrm{C}^{*}$-algebras. This is most prominently witnessed by A. Toms' counterexample in [32], where the author exhibits a simple, separable, unital, nuclear, stably finite $\mathrm{C}^{*}$-algebra $A$ that has the same Elliott invariant as $A \otimes U$ for any UHF-algebra $U$. Yet, the said algebras are not isomorphic since the Cuntz semigroup of $A$ fails to be almost unperforated. In particular, $A$ does not absorb the Jiang-Su algebra $\mathcal{Z}$ tensorially (a condition referred to as $\mathcal{Z}$-stability).

The exact relationship between almost unperforation and $\mathcal{Z}$-stability is one of the ingredients in the well-known Toms-Winter conjecture, that treats the situation of simple, separable, unital, nuclear, and infinite dimensional $\mathrm{C}^{*}$-algebras (see, for example, [33, Remark 3.5], [39, Conjecture 0.1], and [41, Conjecture 9.3]). For these $\mathrm{C}^{*}$-algebras, part of this conjecture predicts that almost unperforation in the Cuntz semigroup and tensorial absorption of the Jiang-Su algebra are equivalent conditions. Following the work of many authors, this equivalence is now known to hold in the case where the tracial simplex is a Bauer simplex whose extreme boundary has finite covering dimension. More concretely, that $\mathcal{Z}$-stability implies almost unperforation holds in full generality (see [29]). The converse follows from the (independent) results in [20], 31] and [34] (see also [21]). Moreover, in some particular cases, a computation of the Cuntz semigroup of $A \otimes \mathcal{Z}$ has been carried out (see e.g. [11, 10, 16]).

Date: October 17, 2016.

2010 Mathematics Subject Classification. Primary 06B35, 15A69, 46L05. 46L35, 46L80, Secondary 18B35, 19K14, 46L06,

Key words and phrases. Cuntz semigroup, tensor product, $C^{*}$-algebra.

R.A. and F.P. were partially supported by grants DGI MICIIN MTM2011-28992-C02-01 and MINECO MTM2014-53644-P; H.P. was supported by the DFG (SFB 878). Part of the results in this paper were obtained while the second author was attending the program Classification of operator algebras: complexity, rigidity and dynamics at the Mittag-Leffler Institute, January-April 2016. 
Albeit possessing this knowledge on the structure of the Cuntz semigroup of a $\mathcal{Z}$-stable algebra, the computation of $\mathrm{Cu}(A \otimes \mathcal{Z})$, given $\mathrm{Cu}(A)$, remains open in the general case. Our study concerns structural properties of $\mathrm{Cu}(A \otimes \mathcal{Z})$ for $\mathrm{C}^{*}$-algebras with low rank. We restrict our investigation to $\mathrm{C}^{*}$-algebras with real rank zero, as for such algebras the Cuntz semigroup is algebraic (that is, the subsemigroup of the so called compact elements is dense; see [4]). In fact, as shown in [14], if $A$ is a $\mathrm{C}^{*}$-algebra with stable rank one, then $A$ has real rank zero precisely when $\mathrm{Cu}(A)$ is algebraic. Towards a successful computation of $\mathrm{Cu}(A \otimes \mathcal{Z})$ when $A$ has real rank zero, the following question naturally arises: If $A$ is a $\mathrm{C}^{*}$-algebra of real rank zero (with no elementary subquotient), is then $A \otimes \mathcal{Z}$ of real rank zero? In terms of the Cuntz semigroup, a related question is whether $A$ having real rank zero implies that $\mathrm{Cu}(A \otimes \mathcal{Z})$ is algebraic.

We note that this question has a positive answer in the simple case. Indeed, let $A$ be a unital, simple, exact, non-type I $\mathrm{C}^{*}$-algebra. Then $A \otimes \mathcal{Z}$ is either purely infinite (simple) or else it has stable rank one, by the results in [29. In the first case, it is well known that $A \otimes \mathcal{Z}$ has real rank zero. In the second case, if $A$ has further real rank zero, then the image of $\mathrm{K}_{0}(A)$ in the space $\operatorname{Aff}(T(A))$ of real valued, affine continuous functions on the trace simplex is dense (see [5] and [24]). Since, as groups, $\mathrm{K}_{0}(A) \cong \mathrm{K}_{0}(A \otimes \mathcal{Z})$ and also $T(A) \cong T(A \otimes \mathcal{Z})$, this density condition is also satisfied by $A \otimes \mathcal{Z}$. Then [29, Theorem 7.2] yields that $A \otimes \mathcal{Z}$ has real rank zero.

A natural approach to answering the question of whether the real rank of $A \otimes \mathcal{Z}$ is zero if $A$ has real rank zero and no elementary subquotients, consists of studying it in the abstract setting of semigroups in the category $\mathrm{Cu}$, as defined in 14. (see below for the precise definition). This can be carried out in (at least) two ways. The first one consists of identifying the subsemigroup of compact elements in the Cuntz semigroup of $A \otimes \mathcal{Z}$. To this end, we shall mostly focus on (residually) stably finite $\mathrm{C}^{*}$-algebras $A$, as in this setting the said subsemigroup agrees with $\mathrm{V}(A)$, the Murray-von Neumann semigroup of projections. In the simple case, the computation of $\mathrm{V}(A \otimes \mathcal{Z})$ is carried out in [17] in the form of an almost unperforated subsemigroup of the Grothendieck group of $\mathrm{V}(A)$. This, of course, uses (stable) cancellation of projections of $A \otimes \mathcal{Z}$ in an essential way, which is possible because the stable rank of a finite, simple $\mathcal{Z}$-stable $\mathrm{C}^{*}$-algebra is one (29]). In the non-simple setting, however, we only know that $\mathrm{V}(A \otimes \mathcal{Z})$ is separative (see [18). To compensate, we bring in an additional and sufficient condition on the $\mathrm{C}^{*}$-algebra $A$ that ensures cancellation for $A \otimes \mathcal{Z}$. This condition is termed cancellation into ideals, which holds vacuously in the simple case and potentially more widely in general. Under this additional assumption and assuming residually stably finiteness, we can then extend the computation of $\mathrm{V}(A \otimes \mathcal{Z})$ from [17] to the non-simple setting.

A second way of approaching the question of when the real rank of $A \otimes \mathcal{Z}$ is zero consists of studying the relationship of $\mathrm{Cu}(A \otimes \mathcal{Z})$ with $\mathrm{Cu}(A)$ and $\mathrm{Cu}(\mathcal{Z})$. The first two authors, in collaboration with $\mathrm{H}$. Thiel, initiated in [4] the investigation of the Cuntz semigroup of tensor products of $\mathrm{C}^{*}$-algebras by introducing and studying the tensor product $\otimes_{\mathrm{Cu}}$ in the category $\mathrm{Cu}$. Denoting $Z=\mathrm{Cu}(\mathcal{Z})$, a verification of a semigroup analogue of part of the Toms-Winter conjecture is shown to hold in [4, Theorem 7.3.11]: A semigroup $S$ in $\mathrm{Cu}$ is almost unperforated and almost divisible if and only if $S \cong$ $S \otimes_{\mathrm{Cu}} Z$. It follows that, if the Toms-Winter conjecture holds true, then the condition $\mathrm{Cu}(A) \cong \mathrm{Cu}(A) \otimes_{\mathrm{Cu}} Z$ is equivalent to $A \cong A \otimes \mathcal{Z}$.

Given $\mathrm{C}^{*}$-algebras $A$ and $B$, of which at least one is nuclear, it was proved in [4] that there is a natural map $\mathrm{Cu}(A) \otimes_{\mathrm{Cu}} \mathrm{Cu}(B) \rightarrow \mathrm{Cu}(A \otimes B)$. This map was shown to be an isomorphism when one of the algebras is either an AF-algebra, or a nuclear $\mathcal{O}_{\infty}$-stable 
algebra ([4, Sections 6,7]). It remains an open problem to decide when the above map is an isomorphism. (See [4, Problem 6.4.11]; it is known this is not the case in general, as observed in [4, 6.4.12].) Still, it is natural to consider the following question: Given a $\mathrm{C}^{*}$-algebra $A$ of real rank zero with no elementary subquotient, is $\mathrm{Cu}(A) \otimes_{\mathrm{Cu}} \mathrm{Cu}(\mathcal{Z})$ also algebraic? This can be phrased in the more general setting of the category $\mathrm{Cu}$ as follows: If $S \in \mathrm{Cu}$ is algebraic, when does it follow that $S \otimes_{\mathrm{Cu}} Z$ is also algebraic? We show here that, if $S$ is algebraic and almost divisible, then $S \otimes_{\mathrm{Cu}} Z$ is algebraic, suggesting that indeed under reasonable assumptions, the real rank of $A \otimes \mathcal{Z}$ is zero whenever the real rank of $A$ is zero.

In the abstract setting, this question is related to two problems posed in [4]. The first of these problems ([4, Problem 7.3.13]) focuses on the semigroup analogue of the natural embedding $j: A \rightarrow A \otimes \mathcal{Z}$, which induces a map $\mathrm{Cu}(j): \mathrm{Cu}(A) \rightarrow \mathrm{Cu}(A \otimes \mathcal{Z})$. This factorises as

$$
\mathrm{Cu}(A) \rightarrow \mathrm{Cu}(A) \otimes_{\mathrm{Cu}} \mathrm{Cu}(\mathcal{Z}) \rightarrow \mathrm{Cu}(A \otimes \mathcal{Z}),
$$

and thus it is natural to ask, at the semigroup level, in which way $\mathrm{Cu}(A)$ sits in $\mathrm{Cu}(A) \otimes_{\mathrm{Cu}} \mathrm{Cu}(\mathcal{Z})$. More concretely, given $S \in \mathrm{Cu}$, is it possible to characterize when $x \otimes 1 \leq y \otimes 1$ in $S \otimes_{\mathrm{Cu}} Z$ ? In the same vein, a natural subproblem asks to characterize how $\mathrm{V}(A)$ sits in $\mathrm{V}(A) \otimes 1$, seen as a subsemigroup of $\mathrm{Cu}(A) \otimes_{\mathrm{Cu}} \mathrm{Cu}(\mathcal{Z})$.

The second problem $([4,7.3 .14])$ aims at measuring how far is $\mathrm{Cu}(A) \otimes_{\mathrm{Cu}} \mathrm{Cu}(\mathcal{Z})$ from being the Cuntz semigroup of a $\mathrm{C}^{*}$-algebra. It is known that $\mathrm{Cu}(A)$ always satisfies two additional axioms than the ones originally used to define the category $\mathrm{Cu}$. The first one is referred to as the axiom of almost algebraic order, or also axiom (O5), and indeed can be thought of as a deformation of the algebraic order (see [30]). The second one is referred to the axiom of almost Riesz decomposition, or also axiom (O6), and it is a version of the Riesz decomposition property in an ordered semigroup; see [27]. (See below for the precise formulations of these axioms.) It is also known that, if $A$ has stable rank one, $\mathrm{Cu}(A)$ has the so called property of weak cancellation. Thus specifically, if $S \in \mathrm{Cu}$, we ask whether axioms (O5), (O6) or weak cancellation pass from $S$ to $S \otimes_{\mathrm{Cu}} Z$.

Building on the work in [4, we provide partial answers to the above questions. For an algebraic semigroup $S \in \mathrm{Cu}$ satisfying (O5), (O6), weak cancellation and almost divisibility, we completely characterize when $x \otimes 1 \leq y \otimes 1$ in $S \otimes_{\mathrm{Cu}} Z$ in terms of comparison of two successive (additive) powers of $x$ and $y$. This relies on a similar characterization we obtain in purely algebraic tensor products.

Our results on the last question above yield a connection of almost unperforation with axiom (O5): If $S$ is an algebraic, almost divisible Cu-semigroup satisfying (O5), and such that the subsemigroup of compact elements is separative, then $S \otimes_{\mathrm{Cu}} Z$ satisfies (O5) if and only if $S$ is almost unperforated. For a simple, non-type I $\mathrm{C}^{*}$-algebra $A$ of real rank zero and stable rank one, this results in the equivalence of $\mathrm{Cu}(A)$ being almost unperforated with $\mathrm{Cu}(A) \otimes_{\mathrm{Cu}} \mathrm{Cu}(\mathcal{Z})$ satisfying (O5). In turn, this is also equivalent to having $\mathrm{Cu}(A) \cong \mathrm{Cu}(A \otimes \mathcal{Z})$. If $A$ has, further, locally finite nuclear dimension, the latter is equivalent to $A \cong A \otimes \mathcal{Z}$, by [40, Corollary 7.4].

The paper is organized as follows. Sections 2 and 3 are devoted to discuss cancellation, divisibility and perforation properties in positively ordered semigroups, with some applications to $\mathrm{Cu}$-semigroups and $\mathrm{C}^{*}$-algebras. We move on in Section 4 to study almost unperforated semigroups and, in particular, embeddings of algebraically ordered semigroups into almost unperforated cones. In Section 5 we discuss the order of tensor products of algebraically ordered semigroups with the Cuntz semigroup of $\mathcal{Z}$. Finally, 
Section 6 contains the applications of the previous sections to $\mathrm{Cu}$-semigroups and $\mathrm{Cuntz}$ semigroups of $\mathrm{C}^{*}$-algebras of real rank zero, including answers to the above questions.

\section{Preliminaries}

The purpose of this section is to recall various definitions concerning positively ordered semigroups that we shall be using throughout.

1.1 (Positively ordered semigroups). All semigroups in this paper are commutative, written additively, and have a neutral element, that we shall denote by 0 .

Recall that a positively ordered semigroup is a semigroup $M$ with a translation invariant partial order $\leq$ such that $0 \leq x$ for all $x \in M$ (see [36] and also [4, B.2.1]). Notice that such a semigroup will always be conical, that is, $x+y=0$ if and only if $x=y=0$.

If $M$ is just a semigroup, then $M$ can be equipped with the algebraic ordering: $x \leq_{\text {alg }} y$ provided there is $z \in M$ with $x+z=y$. Note that $0 \leq_{\text {alg }} x$ for all $x \in M$ and that $\leq_{\text {alg }}$ is clearly translation invariant, though the algebraic ordering might not necessarily be a partial order. If $M$ is finite in the sense that $x+y=x$ implies $y=0$, then $\left(M, \leq_{\text {alg }}\right)$ is also partially ordered. Therefore the projection semigroup $\mathrm{V}(A)$ of a stably finite $\mathrm{C}^{*}$ algebra $A$ is, equipped with the order induced from Murray-von Neumann comparison of projections, a positively ordered semigroup. Since it will be clear from the context, we will not generally use the notation $\leq_{\text {alg }}$ to refer to the algebraic order. Notice that the order in any positively ordered semigroup always extends $\leq_{\text {alg. }}$.

1.2 (order ideals and quotients). Recall that a subsemigroup $I$ of a positively ordered semigroup $M$ is an order ideal provided that $x \leq y$ with $y \in I$ entails that $x$ is an element of $I$. Given $x \in M$, the order ideal generated by $x$ will be denoted by $I(x)$ and will be termed a principal ideal. Observe that $I(x)=\{y \in M \mid y \leq n x$ for some $n \in \mathbb{N}\}$. As is customary, $M$ is said to be simple in case the only order ideals are the trivial ones.

In case $M$ is given the algebraic order, then a subsemigroup $I$ is an order ideal provided $x+y$ is an element of $I$ precisely when $x$ and $y$ are. The ideal generated by $x$ can in this situation be described as $I(x)=\{y \in M \mid y+z=n x$ for some $z \in M$ and $n \in \mathbb{N}\}$.

Recall that the quotient $M / I$ of a positively ordered semigroup $M$ by an order ideal $I$ is defined as $M / \sim$ where $\sim$ is the congruence given by: $x \sim y$ if and only if $x+v=y+w$ with $v, w \in I$. Denote the classes of $M / I$ by $[x]$ for $x \in M$. This new semigroup can be equipped with the translation invariant preorder $[x] \leq[y]$ provided $x \leq y+v$, for $v \in I$. If $M$ is algebraically ordered, then $M / I$ is always conical, but it need not be partially ordered. For general, positively ordered semigroups, the notion of finiteness may be adjusted to say $x+y \leq y$ only when $x=0$. For conical, algebraically ordered semigroups, this agrees with our definition of finiteness given in 1.1

We shall often require this stronger finiteness condition and then say that $M$ is strongly finite provided $M / I$ is finite for all order ideals $I$. (In some contexts, this condition is referred to as $M$ being residually finite.) If $M$ is algebraically ordered and strongly finite, then all of its quotients are partially ordered.

The following elementary fact will be repeatedly used in the sequel, hence we record it for future reference.

Lemma 1.3. Let $M$ be a strongly finite, positively ordered semigroup. If for $x, y, z \in M$,

$$
x+z \leq y+z,
$$

then $I(x) \subseteq I(y)$. 
Proof. Let $I=I(y)$ be the order ideal generated by $y$. Then in $M / I$ we have $[z]+[x] \leq$ $[z]$. By the finiteness assumption on $M$, the element $x$ lies in $I$.

1.4 (The category $\mathrm{Cu}$ ). Given elements $x, y$ in a positively ordered semigroup $S$, we say that $x$ is compactly contained in $y$, in symbols $x \ll y$, if whenever $\left(z_{n}\right)_{n}$ is an increasing sequence in $S$ for which the supremum exists and which satisfies $y \leq \sup _{n} z_{n}$, then there exists $k \in \mathbb{N}$ with $x \leq z_{k}$.

As mentioned in the introduction, the category $\mathrm{Cu}$ was introduced in [14]. The objects are positively ordered semigroups $S$ subject to four axioms:

(O1) Every increasing sequence in $S$ has a supremum;

(O2) Any element $x \in S$ is the supremum of a sequence $\left(x_{n}\right)$ such that $x_{n} \ll x_{n+1}$ for all $n$;

(O3) The relation « is compatible with addition;

(O4) Suprema and addition are compatible.

We refer to an object $S \in \mathrm{Cu}$ as an (abstract) Cu-semigroup. Recall that the Cuntz semigroup $\mathrm{Cu}(A)$ of a $\mathrm{C}^{*}$-algebra $A$ is defined as

$$
\mathrm{Cu}(A)=(A \otimes K)_{+} / \sim
$$

where $\sim$ is the antisymmetrization of the subequivalence defined by $a \precsim b$ if and only if $a=\lim _{n} x_{n} b x_{n}^{*}$ for some sequence $\left(x_{n}\right)_{n}$. It was shown in [14] that $\mathrm{Cu}(A) \in \mathrm{Cu}$ for every $\mathrm{C}^{*}$-algebra $A$.

Ideals of $\mathrm{Cu}$-semigroups are order-ideals as in 1.2 that are further closed under suprema of increasing sequences. The quotient of a Cu-semigroup $S$ by an order ideal $I$ is defined also as in 1.2, except that the order is antisymmetrized, and thus $S / I$ is always positively ordered. In fact, $S / I$ becomes an object in $\mathrm{Cu}$ (see [4, Lemma 5.1.2]).

Recall from [4, 5.2.2] that a $\mathrm{Cu}$-semigroup is stably finite provided $x+y=y$ only when $x=0$ for any $y$ such that there is $\tilde{y} \in S$ with $y \ll \tilde{y}$. We will then say here that $S$ is strongly finite when $S / I$ is stably finite for any ideal $I$ of $S$.

1.5 (Preminimality). Not all semigroups in this paper will be algebraically ordered, namely the Cuntz semigroup of a $\mathrm{C}^{*}$-algebra rarely is. The following concept is then useful:

A positively ordered semigroup $M$ is preminimal if, whenever $x, y, v \in M$ satisfy $x+v \leq y+v$ and $v \leq w$, for $w \in M$, then $x+w \leq y+w$ (see [35, Definition 1.2]). Of course, any algebraically ordered semigroup is automatically preminimal.

For $\mathrm{Cu}$-semigroups (even those that come from $\mathrm{C}^{*}$-algebras), this will be a rare condition to be satisfied. For example, $Z:=\mathrm{Cu}(\mathcal{Z})$ is not preminimal. To check this, identify $Z$ with $\mathbb{N}_{0} \sqcup(0, \infty]$, and denote as $1^{\prime}$ the unit in $(0, \infty]$. We now have $1^{\prime} \leq 1$ and also $1+1^{\prime} \leq 1^{\prime}+1^{\prime}$, but $2 \not \leq 1^{\prime}+1$.

The following notion, close to preminimality, is much more frequent. Let us say that a Cu-semigroup $S$ is weakly preminimal if whenever $x+z \ll y+z$ and $z \leq w$, we have $x+w \leq y+w$.

Recall that a Cu-semigroup $S$ satisfies (O5) (the axiom of almost algebraic order) if, whenever $x+z \leq y$ in $S$ and $x^{\prime} \ll x, z^{\prime} \ll z$, then there is $w \in S$ with $z^{\prime} \leq w$ and $x^{\prime}+w \leq y \leq x+z$. For any $\mathrm{C}^{*}$-algebra $A$, the semigroup $\mathrm{Cu}(A)$ satisfies (O5) (see 4, Definition 4.1, Proposition 4.7], and also [30, Lemma 7.1]).

As shown in [4, Lemma 5.6.7], any Cu-semigroup satisfying (O5) is weakly preminimal. In particular, $\mathrm{Cu}(A)$ is weakly preminimal for any $\mathrm{C}^{*}$-algebra $A$. 
The exact relationship between these two concepts is clarified if we consider algebraic $\mathrm{Cu}$-semigroups. In order to make the connection precise, recall that an element $x$ in a Cu-semigroup is compact provided $x \ll x$. The subsemigroup of compact elements of $S$ is denoted by $S_{c}$. Recall from [4] (see also [15]) that a Cu-semigroup is algebraic provided that every element of $S$ is the supremum of an increasing sequence of compact elements. If $A$ is a stably finite $\mathrm{C}^{*}$-algebra, $\mathrm{V}(A)$ can be identified with the subsemigroup of compact elements in $\mathrm{Cu}(A)$ (see, for example, [9]).

Lemma 1.6. Let $S$ be an algebraic $\mathrm{Cu}$-semigroup. Then $S$ is weakly preminimal if and only if $S_{c}$ is preminimal.

Proof. We need only show the "if" condition. Thus assume $x+z \ll y+z$ and $z \leq w$. Choose $z^{\prime} \ll z^{\prime} \ll z$ such that $x+z^{\prime} \ll y+z^{\prime}$. Write $x$ and $y$ as suprema of sequences $\left(x_{n}\right)$ and $\left(y_{n}\right)$ of compact elements, respectively. We have $x_{n}+z^{\prime} \leq y_{n}+z^{\prime}$ for sufficiently large $n$. Choose an increasing sequence $\left(w_{m}\right)$ of compact elements with suprema $w$. Since $z^{\prime} \ll w$, there is $m_{0}$ such that $z^{\prime} \leq w_{m}$ whenever $m \geq m_{0}$. Apply preminimality of $S_{c}$ to conclude $x_{n}+w_{n} \leq y_{n}+w_{n}$ for suffciently large $n$. Taking suprema we obtain $x+w \leq y+w$, as desired.

\section{Cancellation conditions}

In this section we analyse various notions of cancellation for semigroups associated to $\mathrm{C}^{*}$-algebras, and introduce the much weaker condition of cancellation into ideals.

2.1 (Separativity). There is no doubt that cancellation is a very useful condition that helps analysing the structure of a semigroup. For $\mathrm{V}(A)$, it is automatic when $A$ has stable rank one. For general posivitely ordered semigroups, full cancellation might not be present, although there are weaker forms that we define below. We also need to notice that, in this general context, $M$ is cancellative if $x+z=y+z$ implies $x=y$ and $M$ is order-cancellative if $x+z \leq y+z$ implies $x \leq y$. For algebraically ordered semigroups these two notions of cancellation agree.

A semigroup $M$ is separative if $2 x=x+y=2 y \Longrightarrow x=y$, for $x, y \in M$ (see, for example, [13]). In [5] separativity is shown to be equivalent to a number of properties for algebraically ordered semigroups, one of them being that, if $x, y, z$ are elements in $M$ such that $x+z=y+z$ and $z$ belongs to $I(x), I(y)$, then $x=y$.

In case $M$ is positively ordered, then we say that $M$ is order-separative if $M$ is preminimal, $M$ is separative as a semigroup, and furthermore $x+y \leq 2 y \Longrightarrow x \leq y$. (See [35, Definition 1.2 and Theorem 1.4].)

We consider now a version of this concept, that somewhat sits between strong separativity (in the sense of [22]) and separativity:

We say that $M$ is nearly separative if $M$ is preminimal and $2 x \leq x+y \leq 2 y \Longrightarrow$ $x \leq y$. (This notion was termed weak separativity in [4, but as we will see below it is not really a weakening of the concept of separative cancellation.)

Observe that a nearly separative, positively ordered, semigroup is necessarily separative. In particular, for a partially ordered semigroup, near unperforation as will be defined below implies separativity.

Lemma 2.2. Let $M$ be a positively ordered semigroup. Consider the following conditions:

(i) $M$ is nearly separative.

(ii) $x+z \leq y+z$ with $z \in I(x)$ and $z \in I(y)$ implies $x \leq y$. 
(iii) $x+2 z \leq y+2 z$ implies $x+z \leq y+z$.

Then (i) $\Longleftrightarrow$ (ii) $\Longrightarrow$ (iii).

Proof. Assume (i). To prove (iii), assume $x+2 z \leq y+2 z$. It follows easily that $2(x+z) \leq(x+z)+(y+z) \leq 2(y+z)$, whence (i) implies $x+z \leq y+z$.

To show that (ii) holds also assuming (i), we use that already (iii) above holds. Suppose $x+z \leq y+z$ with $z \in I(x), z \in I(y)$. There is $k \in \mathbb{N}$, that we can take to be a power of 2 , such that $z \leq k x$ and also $z \leq k y$. By preminimality, it follows that $x+k x \leq y+k x$ and hence, applying condition (iii) repeatedly, we obtain $2 x \leq x+y$. Likewise, we obtain $x+y \leq 2 y$. Now condition (i) implies $x \leq y$.

That (ii) implies (i) is easy. We only need to notice that if $2 x \leq x+y \leq 2 y$, then $x+x \leq x+y$ with $x \leq 2 x \leq 2 y$. It is also easy to verify that (ii) implies $M$ is preminimal.

Remark 2.3. The proof of Lemma 2.2 shows that (i) $\Longrightarrow$ (ii) for any order-separative semigroup.

Observe also that an (order-)separative semigroup is, when equipped with the algebraic order, automatically nearly separative if it is finite. (This follows easily from condition (ii) in the above Lemma.) Thus, this notion is of relevance for positively ordered semigroups whose order is not algebraic.

Recall that a Cu-semigroup $S$ has weak cancellation provided $x+z \ll y+z$, for $x, y, z \in S$, implies $x \ll y$. This is always satisfied for $\mathrm{Cu}(A)$ whenever $A$ has stable rank one ([30, Theorem 4.3], and also [28]). For Cu-semigroups, it is convenient that the notion of separativity is adjusted as follows: a Cu-semigroup $S$ is weakly separative if, whenever $x+z \ll y+z$ with $x, y, z \in S$ and $z \in I(x), I(y)$, it follows that $x \ll y$. Also, $S \in \mathrm{Cu}$ will be nearly separative if $S$ is weakly preminimal and $2 x \leq x+y \leq 2 y$ implies $x \leq y$.

Lemma 2.4. Let $S$ be a Cu-semigroup. If $S$ is nearly separative then $S$ is weakly separative.

Proof. We know that condition (iii) in Lemma 2.2 holds (using the same proof). If $x+z \ll y+z$ with $z \in I(x), I(y)$, then there is $z^{\prime} \ll z$ and $y^{\prime} \ll y$ such that $x+z^{\prime} \ll y^{\prime}+z^{\prime}$ and $z^{\prime} \leq 2^{n} x, z^{\prime} \leq 2^{n} y^{\prime}$ for some $n$. Arguing as in Lemma 2.2, using weak preminimality instead of just preminimality, we obtain $x \leq y^{\prime} \ll y$.

Corollary 2.5. Let $S$ be an algebraic $\mathrm{Cu}$-semigroup.

(i) $S$ is weakly separative if and only if $S_{c}$ is nearly separative.

(ii) If $S$ satisfies (O5), then $S$ is weakly separative if and only if $S_{c}$ is separative.

Proof. (i): We already know from Lemma 1.6 that $S$ is weakly preminimal precisely when $S_{c}$ is preminimal. If $S$ is weakly separative, it is easy to check that it is also preminimal, and thus $S_{c}$ is preminimal. It is therefore clear that $S_{c}$ is nearly separative.

For the converse, assume that $S_{c}$ is nearly separative and suppose that $x+z \ll y+z$ with $z \in I(x), I(y)$. Arguing as in Lemma 1.6, we obtain sequences of compact elements $\left(x_{n}\right),\left(y_{n}\right)$, whose suprema are $x$ and $y$ respectively, and a compact element $z^{\prime} \leq z$ such that $x_{n}+z^{\prime} \leq y_{n}+z^{\prime}$ for all $n$. Now, as $z^{\prime} \ll z$, there is $k$ with $z^{\prime} \leq k x, k y$, and thus $z^{\prime} \leq k x_{n}, k y_{n}$ for sufficiently large $n$. Since $S_{c}$ is nearly separative, we obtain $x_{n} \leq y_{n}$, and thus $x \leq y$.

(ii): Assuming (O5), $S_{c}$ is algebraically ordered. 
2.6 (Cancellation into ideals). We say that a semigroup $M$ has cancellation into ideals if, whenever $x, y \in M$ are contained in some order ideal $I$ and $x+z=y+z$ for $z \in M$, then there is $v \in I$ such that $x+v=y+v$. Equivalently, $M$ has cancellation into ideals if, whenever we have $x+z=y+z$ for some $x, y, z \in M$, there exists $v$ in the principal ideal $I(x+y)$ such that $x+v=y+v$.

If $M$ is positively ordered, then $M$ has order-cancellation into ideals if, further to the above condition, whenever $x+z \leq y+z$, then $x+v \leq y+v$ for some $v \in I(x+y)$.

Finally, we say that $M$ has strong order-cancellation into ideals if, whenever $x+z \leq$ $y+z$ with $x \in I(y)$, then there is $v \in I(x)$ such that $x+v \leq y+v$.

Note that any form of cancellation into ideals as above is automatically satisfied for simple semigroups.

For a positively ordered, strongly finite semigroup $M$, strong order-cancellation into ideals implies order-cancellation into ideals. Indeed, if $x+z \leq y+z$ with $x, y$ in some order ideal $I$, then $x \in I(y)$ by Lemma 1.3 and we have $I(y) \subseteq I$. But now there is $v \in I(x) \subseteq I$ such that $x+v \leq y+v$. Likewise, if $x+z=y+z$ for $z$ an element in an order ideal $I$, then by the above argument there are elements $v, w \in I$ such that $x+v \leq y+v$ and $y+w \leq x+w$, from which it follows that $x+(v+w)=y+(v+w)$.

Cancellation into ideals is related to full cancellation via the notion of separativity, as we show below:

Proposition 2.7. Let $M$ be a positively ordered preminimal semigroup. Then the following conditions are equivalent:

(i) $M$ is (order-)cancellative.

(ii) (a) $M$ is (order-)separative.

(b) $M$ has (order-) cancellation into ideals.

(c) $M$ is strongly finite.

Moreover, $M$ is order-cancellative if, and only if, it is strongly finite, nearly separative and has strong order-cancellation into ideals.

Proof. It is clear that (i) implies (ii). Assume condition (ii) and let us show that (i) holds. Suppose that $x+z \leq y+z$. Let $I=I(y)$. By Lemma 1.3, $I(x) \subseteq I$. By (b) we may assume that $z$ belongs to $I$, hence there is $k \in \mathbb{N}$ such that $z \leq k y$. Then order-separativity entails $x \leq y$, by [35, Theorem 1.4].

If $x+z=y+z$, then the above argument shows that $x \leq y$ and $y \leq x$, whence $x=y$ as $M$ is partially ordered.

Concerning the second equivalence, we only need to verify the 'if' direction. Thus, assume suppose that $x+z \leq y+z$. Again by Lemma 1.3, $x$ is an element of $I(y)$. By strong order-cancellation into ideals, we can find $v \in I(x)$ such that $x+v \leq y+v$. Finally, Lemma 2.2 implies that $x \leq y$. In case $x+z=y+z$, we argue as in the previous paragraph.

Remark 2.8. In the simple case, Proposition 2.7 states the well-known fact that ordercancellation is equivalent to order-separativity and finiteness.

2.9 (Grothendieck groups). Given a semigroup $M$, we shall denote by $\operatorname{Gr}(M)$ its Grothendieck group that can be preordered by taking as positive cone $\operatorname{Gr}(M)^{+}=\iota(M)$, where $\iota: M \rightarrow \operatorname{Gr}(M)$ is the Grothendieck map. If $M$ is finite, then $\operatorname{Gr}(M)$ with the order just defined is a partially ordered abelian group.

We can also equip $\operatorname{Gr}(M)$ with a preorder defined by taking as positive cone

$$
\operatorname{Gr}(M)^{++}=\{\iota(x)-\iota(y) \mid y \leq x \text { in } M\} .
$$


It is easy to verify that $\left(\operatorname{Gr}(M), \operatorname{Gr}(M)^{++}\right)$is partially ordered whenever $M$ is. In general, $\operatorname{Gr}(M)^{+} \subseteq \operatorname{Gr}(M)^{++}$, and equality occurs if $M$ is algebraically ordered. (Conversely, if $M$ is cancellative, $\operatorname{Gr}(M)^{+}=\operatorname{Gr}(M)^{++}$implies that the order is algebraic.)

The notion of cancellation into ideals for positively ordered semigroups can be expressed, in the language of groups, as follows:

Lemma 2.10. Let $M$ be a positively ordered semigroup. Then

(i) $M$ has cancellation into ideals if, and only if, for every (principal) order ideal I of $M$, the natural map $\operatorname{Gr}(I) \rightarrow \operatorname{Gr}(M)$ is injective.

(ii) $M$ has order-cancellation into ideals if, and only if, for every (principal) order ideal $I$ of $M$, the natural map $\operatorname{Gr}(I) \rightarrow \operatorname{Gr}(M)$ is an order-embedding with respect to the orderings induced by $\operatorname{Gr}(I)^{++}$and $\operatorname{Gr}(M)^{++}$.

Proof. The proof is a straightforward application of the definitions.

This result allows us to translate the notion to $\mathrm{C}^{*}$-algebras, which gives a characterization of the condition in K-theoretic terms via index maps of exact sequences (see below). We say that a $\mathrm{C}^{*}$-algebra $A$ has cancellation into ideals provided its Murrayvon Neumann semigroup $\mathrm{V}(A)$ has the corresponding property. Of course, if $A$ has (stable) cancellation of projections (for example, if $A$ has stable rank one), then $A$ has cancellation into ideals, for in that case $\mathrm{V}(A)$ is a cancellative semigroup.

Recall that, if an ideal $I$ contains a full projection $p$, then $\mathrm{K}_{0}(I)=\mathrm{Gr}(\mathrm{V}(I))$. Indeed, we have

$$
\mathrm{V}(I) \cong \mathrm{V}(I \otimes \mathcal{K}) \cong \mathrm{V}(p I p \otimes \mathcal{K}) \cong \mathrm{V}(p I p) .
$$

Since $\mathrm{K}_{0}(J)=\operatorname{Gr}(\mathrm{V}(J))$ holds for any unital $\mathrm{C}^{*}$-algebra $J$, we get

$$
\mathrm{Gr}(\mathrm{V}(I)) \cong \mathrm{Gr}(\mathrm{V}(p I p)) \cong \mathrm{K}_{0}(p I p) \cong \mathrm{K}_{0}(I)
$$

Also note that if $p$ is a projection in $A \otimes \mathcal{K}$, then the order ideal $I([p]) \cong \mathrm{V}((A \otimes$ $K) p(A \otimes \mathcal{K}))$.

Proposition 2.11. Let $A$ be a unital $C^{*}$-algebra. Then

(i) $A$ has cancellation into ideals if and only if the index map $\delta_{I}: \mathrm{K}_{1}(A / I) \rightarrow \mathrm{K}_{0}(I)$ is zero for all ideals I of A containing a full projection.

(ii) A has order-cancellation into ideals if and only if the natural map $i_{*}: \mathrm{K}_{0}(I) \rightarrow$ $\mathrm{K}_{0}(A)$ is an order-embedding for all ideals $I$ of $A$ containing a full projection.

Proof. (i): Using the six term exact sequence, the index map $\delta_{I}: \mathrm{K}_{1}(A / I) \rightarrow \mathrm{K}_{0}(I)$ is zero for an ideal $I$ of $A$ if and only if the inclusion map of $I$ into $A$ induces an injective map from $\mathrm{K}_{0}(I)$ into $\mathrm{K}_{0}(A)$. By Lemma 2.10, $V(A)$ has cancellation into ideals if and only if for every principal order ideal $I([p])$ of $V(A)$, the natural map $\operatorname{Gr}(I([p])) \rightarrow \operatorname{Gr}(V(A))=\mathrm{K}_{0}(A)$ is injective (where $p$ is a projection in $A \otimes K$ ).

Set $I=(A \otimes \mathcal{K}) p(A \otimes \mathcal{K})^{-}$which clearly contains a full projection, and now the if part of the statement follows from the fact that $I([p]) \cong V(I)$. Conversely, if $\mathrm{V}(A)$ has cancellation into ideals, and $I$ is an ideal of $A$ containing a full projection $p$, the result follows by considering the order ideal $I([p])$ in $V(A)$.

(ii): This is similar to (i).

Proposition 2.12. Let $A$ be a $C^{*}$-algebra of real rank zero. If $A$ has cancellation into ideals, then so does $A \otimes \mathcal{Z}$. 
Proof. Let $\tilde{I}$ be an ideal of $A \otimes \mathcal{Z}$. Then there is an ideal $I$ of $A$ such that $\tilde{I}=I \otimes \mathcal{Z}$, by [7, Lemma 2.13].

Since $A$ has real rank zero, we have $\mathrm{K}_{0}(I)=\mathrm{Gr}(\mathrm{V}(I))$ for any ideal $I$ of $A$ (see, for example, 25]). We can now apply Lemma 2.10 to conclude that the map

$$
\mathrm{K}_{0}(I)=\mathrm{Gr}(\mathrm{V}(I)) \rightarrow \mathrm{Gr}(\mathrm{V}(A))=\mathrm{K}_{0}(A)
$$

is injective. As we have a commutative diagram:

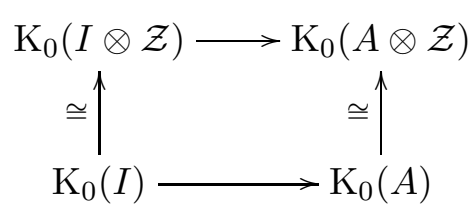

the conclusion follows.

By Proposition 2.11, we have that for a unital $\mathrm{C}^{*}$-algebra $A$, stable cancellation of projections implies that the index map $\delta_{I}$ is zero for all ideals $I$ containing a full projection. However, it is not true that the index map is zero for every ideal of $A$. For an example consider the algebra of continuous functions on the closed unit disk and the ideal consisting of those functions vanishing on the boundary. Hence, cancellation into ideals is both, strictly weaker than stable cancellation, and strictly weaker than the assumption that the index map is zero for all ideals.

It also follows from the argument in Proposition 2.12 that, if $A$ is a $\mathrm{C}^{*}$-algebra of real rank zero, then $A$ has cancellation into ideals precisely when the index map $\delta_{I}$ vanishes for all ideals $I$ of $A$. Equivalently, when the natural map $\mathrm{K}_{1}(A) \rightarrow \mathrm{K}_{1}(A / I)$ is surjective for all ideals $I$.

Recall that a $\mathrm{C}^{*}$-algebra $A$ is termed separative ([5]) if the Murray-von Neumann semigroup $\mathrm{V}(A)$ is separative. Separativity appears quite often in $\mathrm{C}^{*}$-algebra-theory. For example, all known examples of $\mathrm{C}^{*}$-algebras with real rank zero are separative, and it remains an open problem to decide whether this is always the case.

Separativity in a $\mathrm{C}^{*}$-algebra $A$ is known to be equivalent to the following condition, introduced in [8, and termed weak cancellation there: given projections $p$ and $q \in$ $M_{n}(A)$ that generate the same closed two-sided ideal and such that $[p]=[q]$ in $\mathrm{K}_{0}(I)$ are necessarily Murray-von Neumann equivalent. Appealing to [18, Theorem 3.5 and Remark 3.11], it follows that $A \otimes \mathcal{Z}$ is separative for any $\mathrm{C}^{*}$-algebra $A$.

Proposition 2.13. Let $A$ be a stably finite, $\mathcal{Z}$-stable $C^{*}$-algebra of real rank zero. Then $\mathrm{Cu}(A)$ is nearly separative.

Proof. From the comments above we know that $\mathrm{V}(A)$ is separative. We also have $\mathrm{Cu}(A)$ algebraic as $A$ has real rank zero. Then apply Corollary 2.5.

Problem 2.14. If $A$ is a $\mathcal{Z}$-stable $\mathrm{C}^{*}$-algebra, is $\mathrm{Cu}(A)$ nearly separative? Is it weakly separative? (See Problem 3.4.)

According to well established terminology, we say that $A$ is residually stably finite if all quotients of $A$ are stably finite. Since $\mathrm{Cu}(A / I) \cong \mathrm{Cu}(A) / \mathrm{Cu}(I)$ (see [12]), this condition is equivalent to the demand that $\mathrm{Cu}(A)$ is strongly finite. In the case of $\mathrm{V}(A)$, we have the following:

Lemma 2.15. Let $A$ be a $C^{*}$-algebra. If $A$ is residually stably finite, then $\mathrm{V}(A)$ is strongly finite. The converse holds if $A$ has real rank zero. 
Proof. Since the property of being residually stably finite is a stable property, we may assume that $A$ is stable.

For an ideal $J$ of $A$, let $\pi_{J}: A \rightarrow A / J$ denote the natural quotient map. This induces a semigroup map

$$
\rho_{J}: \mathrm{V}(A) / \mathrm{V}(J) \rightarrow \mathrm{V}(A / J)
$$

by $\rho_{J}\left(\pi_{\mathrm{V}(J)}([p])\right)=\left[\pi_{J}(p)\right]$, where $\pi_{\mathrm{V}(J)}: \mathrm{V}(A) \rightarrow \mathrm{V}(A) / \mathrm{V}(J)$ is the natural map.

Next, let $I$ be an order ideal of $\mathrm{V}(A)$, and let

$$
J_{0}:=\{p \in A \mid p \text { is a projection and }[p] \in I\} .
$$

Then $J:=\overline{A J_{0} A}$ is an ideal with $\mathrm{V}(J)=I$.

Suppose that $\bar{x}+\bar{y}=\bar{x}$ in $\mathrm{V}(A) / \mathrm{V}(J)$. Taking representatives $p$ and $q$ for $x$ and $y$ respectively, this means that $\pi_{J}(p) \oplus \pi_{J}(q) \sim \pi_{J}(p)$ in $A / J$. By assumption, this forces $\pi_{J}(q)=0$, and thus $q \in I$, so that $y=0$.

Now assume that $A$ has real rank zero. Then we know from [5, Proposition 1.4] that $\rho_{J}$ is an isomorphism for any ideal $J$ of $A$, and thus $A / J$ is residually stably finite if $\mathrm{V}(A)$ is strongly finite.

Proposition 2.16. Let $A$ be a unital $C^{*}$-algebra of real rank zero that has cancellation into ideals (in particular, this holds if $A$ has real rank zero and stable rank one). Then

$A \otimes \mathcal{Z}$ is residually stably finite $\Longrightarrow \mathrm{V}(A \otimes \mathcal{Z})$ has cancellation.

Proof. By Proposition $2.12 \mathrm{~V}(A \otimes \mathcal{Z})$ has cancellation into ideals. If we further assume that $A \otimes \mathcal{Z}$ is residually stably finite, then by Lemma $2.15 \mathrm{~V}(A \otimes \mathcal{Z})$ is strongly finite. Since by the comments previous to Proposition $2.13 A \otimes \mathcal{Z}$ is always separative, the conclusion follows from Proposition 2.7.

Remark 2.17. It appears plausible that, in general, $A \otimes \mathcal{Z}$ will be stably finite whenever $A$ is. This would improve Proposition 2.16 to show that if $A$ has real rank zero and is residually stably finite, then $A \otimes \mathcal{Z}$ has stable cancellation of projections. Note that, if $A$ has stable rank one and $\mathrm{K}_{1}(I)=0$ for any ideal $I$ of $A$, then already $A \otimes \mathcal{Z}$ has stable rank one ([2, Corollary 1.6]). It is not known (to the authors) whether $A \otimes \mathcal{Z}$ will have stable rank one whenever $A$ has.

\section{DivisibiLITy AND PERFORATION}

Definition 3.1 (Divisibility conditions). Recall that a semigroup $M$ is almost divisible if, for any $x \in M$ and $n \in \mathbb{N}$, there exists an element $z \in M$ such that $n z \leq x \leq(n+1) z$.

Recall also that $M$ is said to be weakly divisible if for any $x \in M$ and $n \in \mathbb{N}$, there are elements $y, z \in M$ such that $x=n y+(n+1) z$.

Lemma 3.2. Let $M$ be an algebraically ordered semigroup. If $M$ is weakly divisible, then it is almost divisible. The converse holds if, moreover, $M$ is cancellative.

Proof. Suppose that $M$ is weakly divisible. Given $x$ and $n$ there are $s, t \in M$ such that $x=n s+(n+1) t$. Then $n(s+t) \leq x \leq(n+1)(s+t)$.

Conversely, suppose that $M$ is cancellative and almost divisible. Let $x \in M$, and $n \in \mathbb{N}$. There is $z \in M$ such that $n z \leq x \leq(n+1) z$. Find $s, t \in M$ such that $n z+t=x$ and $x+s=(n+1) z$. Then $x+z=(n+1) z+t=x+s+t$, and so by cancellation $z=s+t$.

Now $x+s=(n+1) z=(n+1) s+(n+1) t$, whence $x=n s+(n+1) t$, again by cancellation. 
3.3 (Perforation conditions). Given a semigroup $M$ and elements $x, y \in M$, write $x<_{s} y$ provided there is $n \in \mathbb{N}$ such that $(n+1) x \leq n y$. Recall that a semigroup $M$ is termed almost unperforated if $x<_{s} y$ implies $x \leq y$.

As in [4], for a semigroup $M$ we write $x \leq_{p} y$ provided there is $n \in \mathbb{N}$ such that $n x \leq n y$ and $(n+1) x \leq(n+1) y$. We say that $M$ is nearly unperforated if $x \leq_{p} y$ implies $x \leq y$. That near unperforation implies almost unperforation is easy to show (see [4, Proposition 5.6.3]). The converse holds under additional assumptions (see [4] and the discussion below). It is also easy to verify that near unperforation implies near separativity as defined in 2.1 ,

Recall that a partially ordered abelian group $\left(G, G^{+}\right)$is said to be almost unperforated if, whenever $n x,(n+1) x \in G^{+}$, it follows that $x \in G^{+}$. Rørdam proved for an ordered group $\left(G, G^{+}\right)$in [29, Lemma 3.4] that $\left(G, G^{+}\right)$is almost unperforated if and only if $G^{+}$ is almost unperforated as a semigroup. Therefore, we conclude from our observation above that $\left(G, G^{+}\right)$is almost unperforated if and only if $G^{+}$is nearly unperforated.

We recall the following problem, posed in [4]:

Problem 3.4. Let $A$ be a $\mathcal{Z}$-stable $\mathrm{C}^{*}$-algebra. $\mathrm{Is} \mathrm{Cu}(A)$ always nearly unperforated?

As shown by Rørdam in [29, Theorem 4.5], $\mathrm{Cu}(A)$ is almost unperforated if $A$ is $\mathcal{Z}$-stable. A positive answer to the above problem is known in a number of cases, for example, when $A$ has no $\mathrm{K}_{1}$-obstructions. (see [4, 5.6.19]).

The next result implies that, for cancellative, algebraically ordered semigroups, almost unperforation and near unperforation are equivalent properties. Part of the argument is based on [4, Proposition 5.6.12], and we include it for completeness.

Proposition 3.5. Let $M$ be a strongly finite, algebraically ordered semigroup. Then the following statements are equivalent.

(i) $M$ is nearly unperforated and has cancellation into ideals.

(ii) $M$ is almost unperforated and cancellative.

Proof. (i) $\Longrightarrow$ (ii): As already mentioned in 3.3, near unperforation implies almost unperforation. It was proved in [4, Proposition 5.6.3] that, if $M$ is nearly unperforated, then $M$ is nearly separative. Since the order is algebraic, we see that $M$ is actually separative.

Now, let $x, y, z$ be elements in $M$ such that $x+z=y+z$. By Lemma 1.3, $I=I(x)=$ $I(y)$, and thus we may assume that $z$ also belongs to $I$. By separativity, we obtain $x=y$.

(ii) $\Longrightarrow$ (i): Let $x, y$ be elements in $M$ and $n \in \mathbb{N}$ such that $n x \leq n y$ and $(n+1) x \leq$ $(n+1) y$. As the order is algebraic, we can find $c, d \in M$ with $n x+c=n y$ and $(n+1) x+d=(n+1) y$. Hence

$$
(n+1) n x+(n+1) c=(n+1) n y=n(n+1) x+n d .
$$

Cancellation gives us $(n+1) c=n d$ and almost unperforation shows that $c \leq d$. We find $e \in M$ with $c+e=d$. Now,

$$
c+e+(n+1) x+n y=d+(n+1) x+n y=(n+1) y+n x+c .
$$

Cancelling elements we get $e+x=y$, hence $x \leq y$.

Corollary 3.6. Let $M$ be a strongly finite, algebraically ordered semigroup such that $M$ has cancellation into ideals. Then, $M$ is nearly unperforated if and only if $M$ is separative and almost unperforated. 
Proof. Under the assumptions of the corollary all notions of separativity discussed in Section 2.1 agree and, by Proposition 2.7, separativity is equivalent to cancellation. The equivalence then follows from Proposition 3.5.

For simple $\mathrm{Cu}$-semigroups, we have the following

Proposition 3.7. Let $S$ be a simple, stably finite $\mathrm{Cu}$-semigroup that satisfies (O5) and is almost unperforated. Then $S$ is nearly separative if, and only if, $S_{c}$ is separative.

Proof. First note that since $S$ is stably finite and satisfies (O5), $S_{c}$ is nearly separative precisely when it is separative (using, for example, Lemma 2.2).

It is clear that if $S$ is nearly separative, so is $S_{c}$. For the converse, we can use 4, Proposition 5.3.16] to conclude that every element is either compact or non-zero and soft (in the sense of [4, Definition 5.3.1]). Now assume $2 x \leq x+y \leq 2 y$ in $S$. If either $x$ or $y$ are soft, then the argument in [4, Theorem 5.6.10] applies to conclude $x \leq y$. Hence we may assume $x, y \in S_{c}$, in which case near separativity of $S_{c}$ implies $x \leq y$.

We say that a $\mathrm{Cu}$-semigroup $S$ has weak cancellation into ideals if $x+z \ll y+z$ with $x, y$ contained in some ideal $I$, then $x+w \ll y+w$ for some $w \in I$. Observe that this condition is always satisfied in the simple case.

Theorem 3.8. Let $S$ be an algebraic $\mathrm{Cu}$-semigroup that satisfies (O5). Then the following conditions are equivalent:

(i) $S$ is strongly finite, nearly unperforated, and has weak cancellation into ideals.

(ii) $S$ is almost unperforated and weakly cancellative.

Proof. (ii) $\Longrightarrow$ (i) can be easily derived using [4, Proposition 5.6.12], or also Proposition 3.5 .

(i) $\Longrightarrow$ (ii): We know that $S$ is nearly separative, hence $S_{c}$ is a separative semigroup by Corollary 2.5.

Assume $x+z \ll y+z$. Since $S$ is strongly finite, we see that $x \in I(y)$. Using weak cancellation into ideals, we may assume that $z \in I(y)$. Using that $S$ is algebraic, we may reduce to the situation where $x, y$ and $z$ all belong to $S_{c}$ (see, for example, the arguments in Lemma 1.6), with $z \in I(y)$. Find $t \in S_{c}$ and $n \in \mathbb{N}$ such that $x+n y+t=n y+y$. Another use of strong finiteness yields $y \in I(x+t)$. Finally, separativity implies $x+z=y$.

Problem 3.9. Does Theorem 3.8 remain valid without the assumption of $S$ being algebraic?

\section{Constructing almost unperforated cones}

In this section we study the problem of mapping a positively ordered semigroup into an algebraically ordered and almost unperforated semigroup in a universal way. Wehrung showed in [37, Proposition 4.3] that every positively ordered semigroup which is strongly preminimal can be embedded in an algebraically ordered semigroup. We will not define strong preminimality here, but just mention that it is a weakening of separativity. Thus, in particular, every separative semigroup can be embedded in an algebraically ordered semigroup. The difference in our approach resides in that we seek to obtain an additional unperforation condition. 
As we shall see, a natural candidate for this is (the positive cone of) the Grothendieck group of the semigroup, though this will also impose cancellation in the resulting semigroup. We first analyse the setting when the given semigroup is already almost unperforated.

Lemma 4.1. Let $M$ be a strongly finite, algebraically ordered semigroup with cancellation into ideals. If $M$ is nearly unperforated or almost unperforated, then $\operatorname{Gr}(M)^{+}$is almost unperforated.

Proof. Since near unperforation implies almost unperforation, it is enough to assume $M$ is almost unperforated.

Recall that $\iota$ denotes the Grothendieck map $\iota: M \rightarrow \operatorname{Gr}(M)$ and $\operatorname{Gr}(M)^{+}=\iota(M)$. We have to show that $\iota(M)$ is almost unperforated. Suppose there is $n \in \mathbb{N}$ such that $(n+1) \iota(x) \leq n \iota(y)$. Then, as $M$ is algebraically ordered, there are $z, z^{\prime}$ in $M$ such that $(n+1) x+z+z^{\prime}=n y+z$ in $M$. By Lemma 1.3 one gets that $I:=I((n+1) x+$ $\left.z^{\prime}\right)=I(y)$. By cancellation into ideals we can now exchange $z$ for a $\tilde{z} \in I$ such that $(n+1) x+\tilde{z}+z^{\prime}=n y+\tilde{z}$. As in [6. Theorem 2.1.9], we find then some $L \in \mathbb{N}$ such that $L(n+1) x+L z^{\prime}=L n y$. In particular $(L n+1) x \leq L n y$ in $M$. Since $M$ is almost unperforated, $x \leq y$. It follows that $\iota(x) \leq \iota(y)$, which shows almost unperforation of $\iota(M)$, as desired.

Combining this with Lemma 2.15 we immediately obtain:

Corollary 4.2. Let $A$ be a residually stably finite $C^{*}$-algebra that has cancellation into ideals. Then, if $\mathrm{V}(A)$ is almost unperforated so is $\mathrm{K}_{0}(A)^{+}$.

Proposition 4.3. Let $\left(G, G^{+}\right)$be a partially ordered abelian group. Put

$$
G_{\mathrm{au}}^{+}=\left\{x \in G \mid n x,(n+1) x \in G^{+} \text {for some } n\right\} .
$$

Then $G_{\mathrm{au}}^{+}$is a strict cone and $\left(G, G_{\mathrm{au}}^{+}\right)$is almost unperforated. Therefore, $\left(G, G^{+}\right)$is almost unperforated if and only if $G^{+}=G_{\mathrm{au}}^{+}$.

Proof. Let $x, y \in G_{\mathrm{au}}^{+}$. Then, there are $k, l$ such that $k x,(k+1) x \in G^{+}$and $l y,(l+1) y \in$ $G^{+}$. Put $N=3 k l+k+l$. Then $N x=k l x+l(k+1) x+(l+1) k x \in G^{+}$, and likewise $N y \in G^{+}$. Thus $N(x+y) \in G^{+}$. Also, $N+1=2 k l+(k+1)(l+1)$, so that both $(N+1) x$ and $(N+1) y$ belong to $G^{+}$. It follows that $(N+1)(x+y) \in G^{+}$and thus $G_{\mathrm{au}}^{+}$ is a cone.

Suppose now that $x \in G$ satisfies that $\pm x \in G_{\mathrm{au}}^{+}$. Then, there are $k$ and $l \in \mathbb{N}$ such that $k x,(k+1) x \in G^{+}$and also $-l x,-(l+1) x \in G^{+}$. Then, since $\pm k l x \in G^{+}$, we see that $k l x=0$. Arguing similarly, we obtain that $k(l+1) x=(k+1) l x=0$, so that $k x=l x=0$. Since also $(k+1)(l+1) x=0$, it follows that $x=0$.

Finally, suppose that $n x,(n+1) x \in G_{\mathrm{au}}^{+}$. We are to show that $x \in G_{\mathrm{au}}^{+}$. By definition, we can find $k$, and $l \in \mathbb{N}$ such that $k n x,(k+1) n x \in G^{+}$, and $l(n+1) x,(l+1)(n+1) x \in G^{+}$. Put $L=2 k n+n+l+l n$. Then $L x=k n x+(k+1) n x+l(n+1) x \in G^{+}$, and $(L+1) x=2 k n x+(l+1)(n+1) x \in G^{+}$, as desired.

The last part of the statement is clear.

Remark 4.4. Suppose that $G$ is simple as a partially ordered abelian group (that is, every non-zero, positive element is an order-unit). Then

$$
G_{\mathrm{au}}^{+}=\left\{x \in G \mid n x \in G^{+} \backslash\{0\} \text { for some } n\right\} \cup\{0\} .
$$

Indeed, if $n x>0$, then for $N$ large enough $N n x \geq x$, and then $(N n-1) x \in G^{+}$, and also $N n x \in G^{+}$. Conversely, if $n x,(n+1) x \in G^{+}$and $x \neq 0$, then $n x \neq 0$ since otherwise $x>0$, and so $k x>0$ for any $k$. 
4.5. Let $M$ be a finite, positively ordered semigroup. We shall denote $\operatorname{Gr}(M)_{\text {au }}^{+}$to refer to the construction in Proposition 4.3 with respect to the partially ordered abelian group $\left(\operatorname{Gr}(M), \operatorname{Gr}(M)^{+}\right)$.

Since $\operatorname{Gr}(M)^{+} \subseteq \operatorname{Gr}(M)_{\mathrm{au}}^{+}$, we have a natural map $\bar{\iota}: M \rightarrow \operatorname{Gr}(M)_{\mathrm{au}}^{+}$, defined by composing the Grothendieck map $\iota$ with the inclusion. This map is order-preserving when $M$ is algebraically ordered. Note that, if $M$ is cancellative, then $M$ is almost unperforated precisely when $M \cong \operatorname{Gr}(M)_{\text {au }}^{+}$(via $\left.\bar{\iota}\right)$.

The cone $\operatorname{Gr}(M)_{\mathrm{au}}^{+}$defines a preorder $\leq_{\mathrm{au}}$, which is in fact an order by Proposition 4.3. as follows: $x \leq_{\mathrm{au}} y$ if and only if $y-x \in \operatorname{Gr}(M)_{\mathrm{au}}^{+}$.

Observe that, if $N$ is a strict cone in $\operatorname{Gr}(M)$ such that $\iota(M) \subseteq N$ and $(\operatorname{Gr}(M), N)$ is almost unperforated, then $\operatorname{Gr}(M)_{\mathrm{au}}^{+} \subseteq N$. Indeed, if $x \in \operatorname{Gr}(M)_{\mathrm{au}}^{+}$, then $n x,(n+1) x \in$ $\iota(M) \subseteq N$ for some $n$, and then $x \in N$. Note also that one can have strict cones $N$ that contain $\operatorname{Gr}(M)_{\text {au }}^{+}$, yet $(\operatorname{Gr}(M), N)$ is not almost unperforated. For example, take $M=\mathbb{N} \times \mathbb{N}$, so that $\operatorname{Gr}(M)=\mathbb{Z} \times \mathbb{Z}$, and let $N=M+(3,-3) \mathbb{N}$. In this case, $(2,-1) \notin N$, but $2(2,-1), 3(2,-1) \in N$, and so $(\mathbb{Z} \times \mathbb{Z}, N)$ is not almost unperforated.

We may also look at the cone

$$
\operatorname{Gr}(M)^{++}=\{\iota(x)-\iota(y) \mid y \leq x \text { in } M\},
$$

as defined in 2.6, which is a strict cone. The natural map $\iota^{+}: M \rightarrow \operatorname{Gr}(M)^{++}$is orderpreserving, and it is an order-embedding if and only if $M$ is order-cancellative, as is easy to verify. In this situation, we shall denote by $\iota^{+}: M \rightarrow \operatorname{Gr}(M)_{\mathrm{au}}^{++}$the composition of $\iota^{+}$with the inclusion $\operatorname{Gr}(M)^{++} \subseteq \operatorname{Gr}(M)_{\mathrm{au}}^{++}$, which is an order-preserving semigroup morphism.

Since we can construct the cones $\operatorname{Gr}(M)_{\mathrm{au}}^{+}$and $\operatorname{Gr}(M)_{\mathrm{au}}^{++}$, both sitting between $\operatorname{Gr}(M)^{+}$and $\operatorname{Gr}(M)$, it is natural to look at their relative position. By the comments above, as $\left(\operatorname{Gr}(M), \operatorname{Gr}(M)_{\mathrm{au}}^{++}\right)$is almost unperforated (by Proposition 4.3), it follows that $\operatorname{Gr}(M)_{\mathrm{au}}^{+} \subseteq \operatorname{Gr}(M)_{\mathrm{au}}^{++}$in general.

To construct an example where equality does not occur, let $\theta$ be an irrational number, and let $M$ be the subsemigroup of $\mathbb{R}^{+}$generated by positive integer combinations of 1 and $\theta$. Equip $M$ with the natural order, which is clearly not algebraic.

As a semigroup, $M \cong \mathbb{N} \times \mathbb{N}$. Note that $\operatorname{Gr}(M) \cong \mathbb{Z} \times \mathbb{Z}$. With this identification, we have

whereas

$$
\operatorname{Gr}(M)^{+}=\{(a, b)-(c, d) \mid a \geq c, b \geq d\}=\mathbb{N} \times \mathbb{N},
$$

$$
\operatorname{Gr}(M)^{++}=\{(a, b)-(c, d) \mid c+d \theta \leq a+b \theta\} .
$$

Observe that $\operatorname{Gr}(M)_{\text {au }}^{+}=\mathbb{N} \times \mathbb{N}$ and $\operatorname{Gr}(M)^{++} \not \operatorname{Gr}(M)_{\text {au }}^{+}$. This already implies that $\operatorname{Gr}(M)_{\mathrm{au}}^{+} \neq \operatorname{Gr}(M)_{\mathrm{au}}^{++}$, although in this case we have $\operatorname{Gr}(M)_{\mathrm{au}}^{++}=\operatorname{Gr}(M)^{++}$, as is easy to verify.

Proposition 4.6. Let $M$ be a positively ordered semigroup. Then:

(i) If $M$ is nearly unperforated and order-cancellative, then $\operatorname{Gr}(M)^{++}$is nearly unperforated.

(ii) The map $\bar{\iota}^{+}: M \rightarrow \operatorname{Gr}(M)_{\mathrm{au}}^{++}$is an order-embedding if and only if $M$ is ordercancellative and nearly unperforated.

(iii) If $M$ is algebraically ordered, then $\bar{\iota}: M \rightarrow \operatorname{Gr}(M)_{\mathrm{au}}^{+}$is an order-embedding if and only if $M$ is cancellative and almost unperforated.

Proof. (i): Suppose that $M$ is nearly unperforated and order-cancellative. Let $x, y, u, v$ be elements in $M$ such that $y \leq x, v \leq u$ and $n\left(\iota^{+}(x)-\iota^{+}(y)\right) \leq n\left(\iota^{+}(u)-\iota^{+}(v)\right)$ 
for $n \geq n_{0} \in \mathbb{N}$. Then, using order-cancellation on $M$ we obtain $n x+n v \leq n y+n u$ for $n \geq n_{0}$. Near unperforation implies that $x+v \leq y+u$, and thus $\iota^{+}(x)-\iota^{+}(y) \leq$ $\iota^{+}(u)-\iota^{+}(v)$ in $\operatorname{Gr}(M)^{++}$.

(ii): We only have to prove the 'if' direction. Thus assume that $M$ is order-cancellative and nearly unperforated. By (i), we know that $\operatorname{Gr}(M)^{++}$is nearly unperforated, and thus also almost unperforated. Applying Proposition 4.3, we conclude that $\operatorname{Gr}(M)^{++}=$ $\operatorname{Gr}(M)_{\mathrm{au}}^{++}$. Finally, since $M$ is order-cancellative, $\bar{\iota}^{+}=\iota^{+}=$is an order-embedding, as desired.

(iii): This is just a reformulation of (ii) in the algebraically ordered setting.

Remarks 4.7. (a) That near unperforation in $M$ passes to $\operatorname{Gr}(M)^{++}$as in Proposition 4.6 remains true if one starts with a preminimally, positively ordered semigroup $M$ with strong order-cancellation into ideals.

(b) If $M$ is simple, in the sense that every non-zero element is an order-unit, then condition (ii) in Proposition 4.6 can be stated as follows: $\iota(x)<_{\text {au }} \iota(y)$ if and only if $(k+1) x \leq k y$ for some $k$.

Proposition 4.8. Let $M$ be a finite, algebraically ordered semigroup. If $M$ is weakly divisible, then $\operatorname{Gr}(M)_{\mathrm{au}}^{+}$is also weakly divisible.

Proof. Let $x \in \operatorname{Gr}(M)_{\text {au }}^{+}$and $n \in \mathbb{N}$. By definition, there is $k \in \mathbb{N}$ such that $k x=\iota\left(e^{\prime}\right)$, with $e^{\prime} \in M$. Choose $m \in \mathbb{N}$ such that $2 n<m k-1$. Let $e=m e^{\prime}$, and then $m k x=\iota(e)$.

Notice that our choice of $m$ above ensures that $(m k+1) n<(m k-1)(n+1)$. Put $L=(m k+1) n$. As $M$ is almost divisible, there is $z \in M$ such that $L z \leq e \leq(L+1) z \leq$ $(m k-1)(n+1) z$ in $M$. By applying $\bar{\iota}: M \rightarrow \operatorname{Gr}(M)_{\text {au }}^{+}$, and taking into account that $\iota(e)=m k x$, we see that

$$
(m k+1) n \bar{\iota}(z) \leq m k x \leq(m k-1)(n+1) \bar{\iota}(z) .
$$

Now, as $\operatorname{Gr}(M)_{\text {au }}^{+}$is almost unperforated, we obtain that $n \iota(z) \leq x \leq(n+1) \iota(z)$. This shows that $\operatorname{Gr}(M)_{\text {au }}^{+}$is almost divisible and, since it is cancellative, we obtain from Lemma 3.2 that $\operatorname{Gr}(M)_{\mathrm{au}}^{+}$is also weakly divisible.

The assignment $M \mapsto \operatorname{Gr}(M)_{\mathrm{au}}^{++}$is functorial and enjoys a universal property that we now establish.

Theorem 4.9. Let $M$ be a positively ordered semigroup, and let $N$ be an algebraically ordered, cancellative semigroup which is almost unperforated. Then, for any orderpreserving semigroup map $f: M \rightarrow N$ there is a unique semigroup map $\bar{f}: \operatorname{Gr}(M)_{\mathrm{au}}^{++} \rightarrow$ $N$ such that $\bar{f} \circ \bar{\iota}^{+}=f$.

Proof. Identify $N$ with $\operatorname{Gr}(N)^{+}$. By functoriality of the Grothendieck construction, there is a group homomorphism $f_{0}: \operatorname{Gr}(M) \rightarrow \operatorname{Gr}(N)$ such that $f_{0} \circ \iota^{+}=f$.

Define $\bar{f}=\left.f_{0}\right|_{\operatorname{Gr}(M)_{\mathrm{au}}^{++}}$. We need to show that its image is a subset of $N$.

Given $x \in \operatorname{Gr}(M)_{\mathrm{au}}^{++}$, let $n \in \mathbb{N}$ be such that $n x$ and $(n+1) x$ belong to $\operatorname{Gr}(M)^{++}$. Choose $u, v, w, z$ in $M$ with $v \leq u$ and $z \leq w$ in $M$, and such that

$$
n x=\iota^{+}(u)-\iota^{+}(v) \text { and }(n+1) x=\iota^{+}(w)-\iota^{+}(z) .
$$

Rewrite the above equalities as $n x+\iota^{+}(v)=\iota^{+}(u)$ and $(n+1) x+\iota^{+}(z)=\iota^{+}(w)$. Now apply $\bar{f}$ to these equalities to obtain

$$
n \bar{f}(x)+f(v)=f(u) \text { and }(n+1) \bar{f}(x)+f(z)=f(w),
$$


which clearly implies $\bar{f}(x)+f(z)+f(u)=f(w)+f(v)$ in $\operatorname{Gr}(N)$. As $v \leq u$ and $z \leq w$ in $M$, after applying $f$ there are elements $a$ and $b \in N$ with $f(v)+a=f(u)$ and $f(z)+b=f(w)$. Using this and that $\operatorname{Gr}(N)$ is cancellative, we obtain

$$
\bar{f}(x)+a=b .
$$

Now, we also have $(n+1) n x+(n+1) \iota^{+}(v)=(n+1) \iota^{+}(u)$ and $n(n+1) x+\iota^{+}(z)=$ $n \iota^{+}(w)$. Applying $\bar{f}$ to these equalities and using cancellation in $\operatorname{Gr}(N)$ again we get $(n+1) f(u)+n f(z)=(n+1) f(v)+n f(w)$ in $N$. Combine this equality with $f(v)+a=$ $f(u)$ and $f(z)+b=f(w)$ to conclude, using cancellation in $N$, that $(n+1) a=n b$ in $N$. Since $N$ is almost unperforated, we get $a \leq b$, that is, $a+c=b$.

Finally, $\bar{f}(x)+a=b=a+c$, whence $\bar{f}(x)=c$ is an element in $N$, as desired.

The argument we have used also proves that $\bar{f}$ is unique.

Corollary 4.10. Let $M$ and $N$ be algebraically ordered semigroups, with $N$ cancellative and almost unperforated. Then, given a semigroup map $f: M \rightarrow N$, there is a unique semigroup map $\bar{f}: \operatorname{Gr}(M)_{\text {au }}^{+} \rightarrow N$ such that $\bar{f} \circ \bar{\iota}=f$.

Proof. As observed in 4.5, in this case we have $\operatorname{Gr}(M)^{+}=\operatorname{Gr}(M)^{++}$, and thus also $\operatorname{Gr}(M)_{\mathrm{au}}^{+}=\operatorname{Gr}(M)_{\mathrm{au}}^{++}$and $\bar{\iota}=\bar{\iota}^{+}$. The result follows from Theorem 4.9.

Corollary 4.11. Let $M$ and $N$ be positively ordered semigroups. Then, any semigroup map $f: M \rightarrow N$ induces a map $\bar{f}: \operatorname{Gr}(M)_{\mathrm{au}}^{++} \rightarrow \operatorname{Gr}(N)_{\mathrm{au}}^{++}$such that $\bar{f} \circ \bar{\iota}_{M}^{+}=\bar{\iota}_{N}^{+} \circ f$.

Proof. Apply Theorem 4.9 with $\operatorname{Gr}(N)_{\mathrm{au}}^{++}$in place of $N$ and $\vec{\iota}_{N}^{+} \circ f$ in place of $f$.

We will now focus on how the order changes when passing from a $\mathrm{C}^{*}$-algebra $A$ to its $\mathcal{Z}$-stabilisation $A \otimes \mathcal{Z}$, via the natural map $j: A \rightarrow A \otimes \mathcal{Z}$.

Lemma 4.12. Let $A$ be a $C^{*}$-algebra containing a full projection. Then there is a commutative diagram

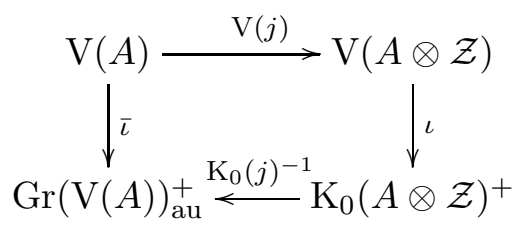

Proof. We of course have a commutative diagram:

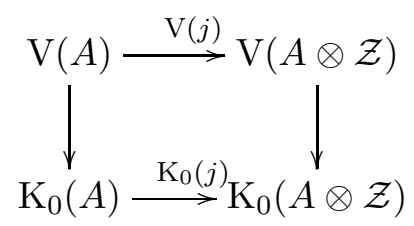

in which the bottom row is an isomorphism and the downward arrows are the obvious ones. Since $A$ has a full projection, $\mathrm{K}_{0}(A)=\mathrm{Gr}(\mathrm{V}(A))$ and we have $\iota(\mathrm{V}(A))=\mathrm{K}_{0}(A)^{+}$. Let $x$ be an element in $\mathrm{K}_{0}(A)$ and suppose that $\mathrm{K}_{0}(j)(x) \geq 0$ in $\mathrm{K}_{0}(A \otimes \mathcal{Z})$. Then, there are relatively prime numbers $m, n$ such that $\mathrm{K}_{0}\left(j_{m, n}\right)(x) \geq 0$ in $K_{0}\left(A \otimes Z_{m, n}\right)$. Arguing exactly as in the proof of [17, Theorem 1.4], we see that $m x \geq 0$ and $n x \geq 0$ in $\mathrm{K}_{0}(A)$.

Now, find $a, b \in \mathbb{Z}$ such that $a m+b n=1$. Writing $a=a_{1}-a_{2}, b=b_{1}-b_{2}$ for $a_{i}, b_{i}$ positive integers, we see that, with $L=a_{2} m+b_{2} n \in \mathbb{N}$, we have that $L+1=a_{1} m+b_{1} n$. Thus $L x$ and $(L+1) x$ both belong to $\mathrm{K}_{0}(A)^{+}$, and this means that $x \in \operatorname{Gr}(V(A))_{\mathrm{au}}^{+}$.

To complete the argument, take $y \in \mathrm{K}_{0}(A \otimes \mathcal{Z})^{+}$. Since $\mathrm{K}_{0}(j)$ is an isomorphism, there is $x \in \mathrm{K}_{0}(A)$ with $\mathrm{K}_{0}(j)(x)=y$, and now the previous paragraph applies to show that in fact $x$ belongs to $\operatorname{Gr}(\mathrm{V}(A))_{\mathrm{au}}^{+}$. 
We now extend [17, Theorem 1] to compute precisely what the positive cone of $\mathrm{K}_{0}(A \otimes$ $\mathcal{Z})$ is in terms of $\mathrm{V}(A)$.

Theorem 4.13. Let $A$ be a $C^{*}$-algebra containing a full projection. Suppose that $A$ is residually stably finite and has cancellation into ideals. Let $j: A \rightarrow A \otimes \mathcal{Z}$ be the natural embedding. Then

(i) $\mathrm{K}_{0}(A \otimes \mathcal{Z})^{+}=j_{*}\left(\operatorname{Gr}(V(A))_{\text {au }}^{+}\right)$and thus is partially ordered and almost unperforated.

(ii) $j_{*}: \mathrm{K}_{0}(A) \rightarrow \mathrm{K}_{0}(A \otimes \mathcal{Z})$ is an isomorphism of ordered groups if and only if $\mathrm{K}_{0}(A)$ is almost unperforated.

Proof. (i). Take $x \in \operatorname{Gr}(V(A))_{\mathrm{au}}^{+}$. By Lemma 4.12, we have to show that $j_{*}(x) \geq 0$ in $\mathrm{K}_{0}(A \otimes \mathcal{Z})$. There is $n \in \mathbb{N}$ such that $n x,(n+1) x \in \mathrm{K}_{0}(A)^{+}$. It follows that $n j_{*}(x)$, $(n+1) j_{*}(x) \in K_{0}(A \otimes \mathcal{Z})^{+}$. But $K_{0}(A \otimes \mathcal{Z})^{+}=G r(V(A \otimes \mathcal{Z}))^{+}$is almost unperforated by [29, Corollary 4.8] and Corollary 4.2, hence it is nearly unperforated as a cancellative almost unperforated semigroup and $j_{*}(x) \geq 0$.

(ii). As $j_{*}$ is already an isomorphism of abelian groups, we have that $j_{*}$ is an isomorphism of ordered groups precisely when $j_{*}\left(\mathrm{~K}_{0}(A)^{+}\right)=\mathrm{K}_{0}(A \otimes \mathcal{Z})^{+}$. By (i), this will happen if and only if $j_{*}\left(\mathrm{~K}_{0}(A)^{+}\right)=j_{*}\left(\operatorname{Gr}(\mathrm{V}(A))_{\mathrm{au}}^{+}\right)$, that is, $\mathrm{K}_{0}(A)^{+}=\operatorname{Gr}(V(A))_{\mathrm{au}}^{+}$. But this is equivalent to saying that $\mathrm{K}_{0}(A)$ is almost unperforated, by Proposition 4.3.

Remark 4.14. Note that the condition assumed on Theorem 4.13 is not necessary. An example to show this is the Toeplitz algebra $\mathcal{T}=C^{*}(s)$, where $s$ is a non-unitary isometry with $1-s s^{*}$ a rank one projection. It is well-known that $\mathcal{T}$ can be equivalently defined through a short exact sequence $0 \rightarrow \mathcal{K} \rightarrow \mathcal{T} \rightarrow C(\mathbb{T}) \rightarrow 0$. We know that $K_{0}(\mathcal{T}) \cong \mathbb{Z}$, with positive cone isomorphic to $\mathbb{N}_{0}$. On the other hand, $\mathrm{K}_{0}(\mathcal{T} \otimes \mathcal{Z})=\mathbb{Z}$ and, since $C(\mathbb{T}, \mathcal{Z})$ has stable rank one, we have that $\mathrm{K}_{0}(C(\mathbb{T}, \mathcal{Z}))^{+}=\mathrm{V}(C(\mathbb{T}, \mathcal{Z})) \cong \mathbb{N}_{0}$. It follows that $\mathrm{K}_{0}(\mathcal{T} \otimes \mathcal{Z})^{+} \cong \mathbb{N}_{0} \cong j_{*}\left(\operatorname{Gr}(\mathrm{V}(\mathcal{T}))_{\text {au }}^{+}\right.$) (but $\mathcal{T}$ is neither stably finite, nor does $\mathrm{V}(\mathcal{T})$ have cancellation into ideals).

Corollary 4.15. Let $A$ be a $C^{*}$-algebra of real rank zero that contains a full projection. Suppose that $A$ is residually stably finite and has cancellation into ideals (in particular, this holds if $A$ has stable rank one). If $A$ is weakly divisible, so is $A \otimes \mathcal{Z}$.

Proof. This is a consequence of Theorem 4.13 and Proposition 4.8.

Corollary 4.16. Let $A$ be a $C^{*}$-algebra that contains a full projection. Suppose that $A$ has real rank zero, cancellation into ideals, and $A \otimes \mathcal{Z}$ is residually stably finite.

Then the map $\mathrm{V}(j): \mathrm{V}(A) \rightarrow \mathrm{V}(A \otimes \mathcal{Z})$ is an order-embedding if and only if $\mathrm{V}(A)$ is almost unperforated and cancellative, if and only if $\mathrm{V}(A)$ is nearly unperforated.

Proof. By Proposition 2.16, $\mathrm{V}(A \otimes \mathcal{Z})$ is a cancellative semigroup, and thus it can be identified with $\mathrm{K}_{0}(A \otimes \mathcal{Z})^{+}$. We know by [29, Corollary 4.8] that $\mathrm{V}(A \otimes \mathcal{Z})$ is always almost unperforated. Thus $\mathrm{V}(A)$ is almost unperforated and cancellative if $\mathrm{V}(j)$ is an order-embedding. Conversely, if $\mathrm{V}(A)$ is cancellative we may apply Theorem 4.13 to get $\mathrm{K}_{0}(A \otimes \mathcal{Z})^{+} \cong \operatorname{Gr}(\mathrm{V}(A))_{\mathrm{au}}^{+}$, and $\mathrm{V}(j)$ is an order-embedding if, further, $\mathrm{V}(A)$ is almost unperforated, by Proposition 4.6.

The last equivalence follows from Proposition 3.5.

Recall from [2] (see also [3]) that a $\mathrm{C}^{*}$-algebra $A$ has no $\mathrm{K}_{1}$-obstructions provided $A$ has stable rank one and $\mathrm{K}_{1}(I)=0$ for every ideal $I$ of $A$. 
Corollary 4.17. Let $A$ be a $C^{*}$-algebra that contains a full projection. If $A$ has no $\mathrm{K}_{1}$-obstructions, then the map $\mathrm{V}(j): \mathrm{V}(A) \rightarrow \mathrm{V}(A \otimes \mathcal{Z})$ is an order-embedding if and only if $\mathrm{V}(A)$ is almost unperforated, if and only if $\mathrm{V}(A)$ is nearly unperforated.

Proof. We know that both $A$ and $A \otimes \mathcal{Z}$ have stable rank one (the latter by [2, Corollary 1.6]), and thus $\mathrm{V}(A)$ and $\mathrm{V}(A \otimes \mathcal{Z})$ are cancellative semigroups. Thus we may identify the latter with $\mathrm{K}_{0}(A \otimes \mathcal{Z})^{+}$, which equals $\operatorname{Gr}(\mathrm{V}(A))_{\text {au }}^{+}$, by Theorem 4.13. Therefore, Proposition 4.6 (iii) implies that $\mathrm{V}(j)$ is an order-embedding exactly when $\mathrm{V}(A)$ is almost unperforated. This, in turn, is equivalent to $\mathrm{V}(A)$ being nearly unperforated by Proposition 3.5.

\section{ORDER IN TENSOR PRODUCTS}

In this section we analyse the map $\mathrm{V}(A) \rightarrow \mathrm{V}(A) \otimes 1_{Z}$ and prove that it encodes the property of near unperforation in $\mathrm{V}(A)$. In the sequel, this result will be transported to the more general setting of $\mathrm{Cu}$-semigroups. As a consequence, and as mentioned in the introduction, we will obtain partial answers to [4, Problems 7.3.13 and 7.3.14].

Much of our discussion below will be carried out for a positively ordered semigroup $M$. Recall that $Z:=\mathrm{Cu}(\mathcal{Z})$ is identified with $\mathbb{N}_{0} \sqcup(0, \infty]$ and that $1_{Z}=\left[1_{\mathcal{Z}}\right]$ corresponds to the compact element $1 \in \mathbb{N}_{0}$.

$\mathbf{5 . 1}$ (Tensor products of positively ordered semigroups). We briefly sketch the construction of the tensor product of positively ordered semigroups, since the concrete form of the partial ordering that can be given to them will be needed below (see also [38, ,4, Appendix B]).

Given $M$ and $N$ positively ordered semigroups, one first constructs the tensor product as conical semigroups. Set $F=\mathbb{N}\left[M^{\times} \times N^{\times}\right]$to be the free abelian semigroup with basis $M^{\times} \times N^{\times}$and whose elements we denote by $a \odot b, a \in M^{\times}, b \in N^{\times}$. Given $f_{0}, g_{0} \in F$ we set $f_{0} \rightarrow^{0} g_{0}$ if $f_{0}=a \odot b$ and $g_{0}=\sum_{i, j} a_{i} \odot b_{j}$ where $a=\sum_{i} a_{i}$ and $b=\sum_{j} b_{j}$. Then, given $f, g \in F$ we set $f \rightarrow g$ if $f=\sum_{k} f_{k}, g=\sum_{k} g_{k}$ and $f_{k} \rightarrow^{0} g_{k}$. Setting $\cong$ as the congruence relation on $F$ generated by $\rightarrow$ and $\leftarrow$, one obtains $M \otimes N=F / \cong$ to be the tensor product of $M$ and $N$ as conical semigroups.

To obtain a partially ordered semigroup, one further considers the relations $f_{0} \leq{ }^{0} g_{0}$ whenever $f_{0}=0$ or $f_{0}=a^{\prime} \odot b^{\prime}, g=a \odot b$ and $a^{\prime} \leq a, b^{\prime} \leq b$. Then set $f \leq \prime g$ whenever $f=\sum_{k} f_{k}, g=\sum_{k} g_{k}$ and $f_{i} \leq{ }^{0} g_{i}$. This defines a preorder relation $\leq$ on $F$ as the additive transitive relation generated by $\cong$ and $\leq^{\prime}$. Thus $f \leq g$ if there exists $n \in \mathbb{N}$ and $f_{k}, f_{k}^{\prime} \in F$ for $k=0, \ldots, n$ such that

$$
f=f_{0} \leq^{\prime} f_{0}^{\prime} \cong f_{1} \leq^{\prime} f_{1}^{\prime} \cong \ldots \cong f_{n} \leq f_{n}^{\prime}=g
$$

Finally, antisymmetrizing this relation $\leq$ we obtain the tensor product $M \otimes N$ in the category of positively ordered semigroups. Let $\omega: F \rightarrow M \otimes N$ be the quotient map, denote the image of the generators by $a \otimes b$ and for general elements $f=\sum_{i} a_{i} \odot b_{i}$ write $\bar{f}:=\omega(f)=\sum_{i} a_{i} \otimes b_{i}$.

Given a positively ordered semigroup $M$, we denote by $M \otimes 1_{Z}$ or $M \otimes 1$ the subsemigroup of $M \otimes Z$ of elements that can be written as $x \otimes 1$ for some $x \in M$. We equip $M \otimes 1$ with the induced order from $M \otimes Z$ and we want to characterize when $x \otimes 1 \leq y \otimes 1$ in terms of the order in $M$. Recall that, for elements $x, y$ in $M$, we write $x<_{s} y$ when there is $n \in \mathbb{N}$ such that $(n+1) x \leq n y$, and $x \leq_{s} y$ provided $x \leq y$ or else $x<_{s} y$.

Lemma 5.2. Let $M$ be a positively ordered semigroup, and let $x, y \in M$. 
(i) If $x \leq_{s} y$, then $x \otimes 1 \leq y \otimes 1$ in $M \otimes 1$.

(ii) If $x \otimes 1 \leq y \otimes 1$ then $x \leq_{p} y$ in $M$.

Proof. Statement (i) can be derived from more general results in [4], but it is nevertheless simple enough to give a self contained argument. First, we may clearly assume that $x, y \neq 0$ and $x<_{s} y$. Suppose $(n+1) x \leq n y$ for some $n \geq 1$ and then

$$
x \otimes 1 \leq x \otimes 1+x \otimes \frac{1}{n}=(n+1) x \otimes \frac{1}{n} \leq n y \otimes \frac{1}{n}=y \otimes 1^{\prime} \leq y \otimes 1 .
$$

We now turn to (ii). Assume $x \otimes 1 \leq y \otimes 1$. By the construction of the tensor product, there exists $m \in \mathbb{N}$ and $f_{k}, f_{k}^{\prime} \in \mathbb{N}\left[M^{\times} \times Z^{\times}\right]$for $k=0, \ldots, m$ such that

$$
x \odot 1=f_{0} \leq^{\prime} f_{0}^{\prime} \cong f_{1} \leq^{\prime} f_{1}^{\prime} \cong \ldots \cong f_{m} \leq f_{m}^{\prime} \cong f_{m+1}=y \odot 1 .
$$

We will see that the elements $f_{i}, f_{i}^{\prime}$ can be chosen of a particular form. To this end, let us introduce some new notation. We denote by $\mathbb{N}^{\prime}$ the set of non compact natural numbers in $Z$. Then, given a prime $p>0$, we consider $\mathbb{N}^{\prime}[1 / p]$ the dense subset of $(0, \infty) \subset Z$ and denote $Z_{p}=\mathbb{N}_{0} \sqcup \mathbb{N}^{\prime}[1 / p] \subset Z$ which can be seen as a discrete version of $Z$.

First suppose $f_{i}, f_{i}^{\prime} \in \mathbb{N}\left[M^{\times} \times\left(\mathbb{N} \sqcup \mathbb{N}^{\prime}\right)^{\times}\right]$. In this case we will prove that $x \leq y$, and it will be enough to prove that whenever $f \leq^{\prime} g, f \rightarrow g$ or $f \leftarrow g$ with $f=\sum_{i=1}^{n} x_{i} \odot n_{i}$, $g=\sum_{j=1}^{m} y_{j} \odot m_{j}, x_{i}, y_{j} \in V$ and $n_{i}, m_{j} \in \mathbb{N} \sqcup \mathbb{N}^{\prime}$, then $\sum x_{i} n_{i} \leq \sum y_{j} m_{j}$.

Suppose $f \leq^{\prime} g$, then $m=n, x_{i} \leq y_{i}, n_{i} \leq m_{j}$ and thus, clearly $\sum_{i} n_{i} \cdot x_{i} \leq \sum_{i} m_{i} \cdot y_{i}$. If $f \rightarrow g$. Then $x_{i}=\sum_{j} x_{i j}, n_{i}=\sum_{k} n_{i k}$ and $g=\sum_{i, j, k} x_{i j} \odot n_{i k}$, and it is clear that $\sum_{i} x_{i} n_{i}=\sum_{i, j, k} x_{i j} n_{i k}$ as desired. Finally, the case $f \leftarrow g$ is similar.

We claim that for any prime $p>0, f_{i}, f_{i}^{\prime}$ can be chosen in $\mathbb{N}\left[M^{\times} \times Z_{p}^{\times}\right]$. Then, multiplying (10) by a certain power $p^{n_{0}}$, we will obtain a chain

$$
\left(p^{n_{0}} x\right) \odot 1=h_{0} \leq^{\prime} h_{0}^{\prime} \cong h_{1} \leq^{\prime} h_{1}^{\prime} \cong \ldots \cong h_{m} \leq h_{m}^{\prime} \cong h_{m+1}=\left(p^{n_{0}} y\right) \odot 1,
$$

where each $h_{i} \in \mathbb{N}\left[M^{\times} \times\left(\mathbb{N} \sqcup \mathbb{N}^{\prime}\right)^{\times}\right]$, and by the previous argument $p^{n_{0}} x \leq p^{n_{0}} y$. Doing this for two different primes $p, q$ yelds $x \leq_{p} y$ as desired.

Let us prove the claim. Set $p>0$ any prime. First, note that by adding trivial relations of the form $\leq^{\prime}$, one can assume that in (1) we have a concatenation of chains of the form $f_{i} \leq^{\prime} f_{i}^{\prime} \rightarrow f_{i+1}$ or $f_{i} \leq^{\prime} f_{i}^{\prime} \leftarrow f_{i+1}$.

Given $f, g \in \mathbb{N}\left[M^{\times} \times Z^{\times}\right]$we will write $f \prec g$ provided $f=\sum_{i=1}^{n} x_{i} \odot \alpha_{i}, g=$ $\sum_{i=1}^{n} y_{i} \odot \beta_{i}$ with $x_{i} \leq y_{i}$ and $\alpha_{i} \ll \beta_{i}$.

We set $g_{0}=x \odot 1 \in \mathbb{N}\left[M^{\times} \times Z_{p}^{\times}\right]$, for which we have $g_{0} \prec f_{0} \leq^{\prime} f_{0}^{\prime} \rightleftarrows f_{1}$, and we will inductively modify (10), by replacing $g_{n} \prec f_{n} \leq^{\prime} f_{n}^{\prime} \rightleftarrows f_{n+1}$, where $g_{n} \in \mathbb{N}\left[M^{\times} \times Z_{p}^{\times}\right]$, with $g_{n} \leq^{\prime} g_{n}^{\prime} \rightleftarrows g_{n+1} \prec f_{n+1}$ with $g_{n}^{\prime}, g_{n+1} \in \mathbb{N}\left[M^{\times} \times Z_{p}^{\times}\right]$.

$$
\left(g_{n} \prec f_{n} \leq^{\prime} f_{n}^{\prime} \rightleftarrows f_{n+1}\right) \rightsquigarrow\left(g_{n} \leq^{\prime} g_{n}^{\prime} \rightleftarrows g_{n+1} \prec f_{n+1}\right)
$$

We first consider the case $g_{n} \prec f_{n} \leq^{\prime} f_{n} \rightarrow f_{n+1}$ with $g_{n} \in \mathbb{N}\left[M^{\times} \times Z_{p}^{\times}\right]$.

Write $g_{n}=\sum_{i=1}^{r} x_{i} \odot \alpha_{i}, f_{n}=\sum_{i=1}^{r} x_{i}^{\prime} \odot \alpha_{i}^{\prime}$ and $f_{n}^{\prime}=\sum_{i=1}^{r} y_{i} \odot \beta_{i}, x_{i} \leq x_{i}^{\prime} \leq y_{i}$ and $\alpha_{i} \ll \alpha_{i}^{\prime} \leq \beta_{i}$. Since $f_{n} \rightarrow f_{n+1}$ we have $y_{i}=\sum_{j=1}^{s_{i}} y_{i j}, \beta_{i}=\sum_{k=1}^{t_{i}} \beta_{i j}$, and $f_{n+1}=\sum_{i=1}^{r} \sum_{j, k} y_{i j} \odot \beta_{i k}$.

Now, if $\beta_{i} \in \mathbb{N}$ (i.e. if it is compact), set $\beta_{i}^{\prime}=\beta_{i}$, note that $\beta_{i k} \in \mathbb{N}$ (also compact) and set $\beta_{i k}^{\prime}=\beta_{i k}$. If $\beta_{i} \notin \mathbb{N}$, then as $\alpha_{i} \ll \alpha_{i}^{\prime} \leq \beta_{i}$, we have $\alpha_{i}<\beta_{i}=\sum_{k=1}^{t_{i}} \beta_{i k}$, and we can choose $\beta_{i k}^{\prime} \in \mathbb{N}^{\prime}[1 / p]$, with $\beta_{i k}^{\prime}<\beta_{i k}$ and such that $\alpha_{i} \leq \beta_{i}^{\prime}:=\sum_{k=1}^{t_{i}} \beta_{i k}^{\prime}$.

Define $g_{n}^{\prime}:=\sum_{i=1}^{r} y_{i} \odot \beta_{i}^{\prime}, g_{n+1}:=\sum_{i, j, k} y_{i j} \odot \beta_{i k}^{\prime}$ and we are done.

Now consider the case that $g_{n} \prec f_{n} \leq^{\prime} f_{n}^{\prime} \leftarrow f_{n+1}$ with $g_{n} \in \mathbb{N}\left[M^{\times} \times Z_{p}^{\times}\right]$. 
We now change notation and write, since $f_{n} \leftarrow f_{n+1}, f_{n+1}=\sum_{i=1}^{r} x_{i} \odot \alpha_{i}$, with $x_{i}=$ $\sum_{j=1}^{s_{i}} z_{i j}, \alpha_{i}=\sum_{k=1}^{t_{i}} \alpha_{i k}$ and such that $f_{n}^{\prime}=\sum_{i} \sum_{j, k} z_{i j} \odot \alpha_{i k}$. Also, $f_{n}=\sum_{i, j, k} w_{i j}^{\prime} \odot \beta_{i k}^{\prime}$ and $g_{n}=\sum_{i, j, k} w_{i j} \odot \beta_{i k}$, with $w_{i j} \leq w_{i j}^{\prime} \leq z_{i j}$ and $\beta_{i k} \ll \beta_{i k}^{\prime} \leq \alpha_{i j}$.

If $\alpha_{i k} \in \mathbb{N}$, set $\alpha_{i k}^{\prime}=\alpha_{i k}$. If $\alpha_{i k} \notin \mathbb{N}$, since $\beta_{i k} \ll \beta_{i k}^{\prime} \leq \alpha_{i k}$, we have $\beta_{i k}<\alpha_{i k}$ and we can choose $\alpha_{i k}^{\prime} \in \mathbb{N}^{\prime}[1 / p]$ such that $\beta_{i k} \leq \alpha_{i k}^{\prime}<\alpha_{i k}$. Setting $\alpha_{i}^{\prime}:=\sum_{i, k} \alpha_{i k}^{\prime} \in \mathbb{N}^{\prime}[1 / p] \sqcup \mathbb{N}$, $g_{n}^{\prime}:=\sum_{i, j, k} z_{i j} \odot \alpha_{i k}$ and $g_{n+1}:=\sum_{i} x_{i} \odot \alpha_{i}^{\prime}$ satisfy the conditions.

By induction, and since $f_{m+1}=y \odot 1$ is already of the desired form, we have $x \odot 1=$ $g_{0} \leq^{\prime} g_{0}^{\prime} \cong g_{1} \leq^{\prime} g_{1}^{\prime} \cong \ldots \cong g_{m} \leq g_{m}^{\prime} \cong g_{m+1}=y \odot 1$, where $g_{i}, g_{i}^{\prime} \in \mathbb{N}\left[M^{\times} \times Z_{p}^{\times}\right]$, which proves the claim and completes the proof.

Note that the converse of (i) is not allways true as the following example testifies.

Example 5.3. Let $N$ be a simple, positively ordered semigroup. Suppose that $N$ has two elements $a, b$ such that $a \not \leq b$, but there is some $n \in \mathbb{N}$ with $(n+1) a \leq n b$ (i.e. $N$ is not almost unperforated). Consider the elements $x=(a, 1), y=(b, 1) \in M:=N \oplus \mathbb{N}$. Clearly, $x \not \leq y$ in $M$, and $(n+1) x \not \leq n y$ for any $n \in \mathbb{N}$. Finally, as $M \otimes Z \cong N \otimes Z \oplus Z$, we see that indeed in this case $x \otimes 1 \leq y \otimes 1$.

We now turn into the question of analysing when $M \otimes 1$ is a nearly unperforated semigroup.

Theorem 5.4. Let $M$ be a positively ordered semigroup. Consider the following conditions:

(i) $M$ is nearly unperforated.

(ii) The map $M \rightarrow M \otimes 1$ is an order-embedding.

(iii) $M$ is almost unperforated.

Then (i) $\Longrightarrow$ (ii) $\Longrightarrow$ (iii). If $M$ is algebraically ordered and cancellative, (iii) $\Longrightarrow$ (i) and all three conditions are equivalent.

Proof. The first part follows from Lemma 5.2. If $M$ is algebraically ordered, cancellative, and almost unperforated, then it is also nearly unperforated by Proposition 3.5.

Remarks 5.5. Using results from [4] that we will discuss in the next section, one can show that $M \otimes 1$ is always almost unperforated. However, as the order is not necessarily algebraic, it does not appear possible to immediately conclude near unperforation.

If $M$ is nearly unperforated and separative the map $M \rightarrow M \otimes 1$ is not only an orderembedding, but an order-isomorphism. Indeed, if $x \otimes 1=y \otimes 1$, then by Lemma 5.2 we have $n x=n y$ and $(n+1) x=(n+1) y$ for some $n \in \mathbb{N}$. Applying separativity we obtain $x=y$. (Of course, assuming only separativity $M$ and $M \otimes 1$ are isomorphic as semigroups.)

A semigroup $M$ is said to be a refinement semigroup if whenever $x_{1}+x_{2}=y_{1}+y_{2}$ in $M$, there are elements $z_{i j} \in M$ for $i=1,2$ such that $x_{i}=z_{i 1}+z_{i 2}$ and $y_{j}=z_{1 j}+z_{2 j}$ for $i, j=1,2$. These semigroups are relevant since $\mathrm{V}(A)$ is always a refinement semigroup for any $\mathrm{C}^{*}$-algebra $A$ of real rank zero.

Definition 5.6. Let $M$ be a positively ordered semigroup. For elements $x, y \in M$, write $x \leq_{d} y$ provided there are elements $x_{i}, y_{i} \in M$, for $i=1,2$ such that $x=x_{1}+x_{2}$, $y=y_{1}+y_{2}$, and $x_{1} \leq y_{1}$, while $x_{2}<_{s} y_{2}$.

Lemma 5.7. Let $M$ be a positively ordered semigroup. Consider the following conditions, for $x, y \in M$ :
(i) $x \leq_{s} y$
(ii) $x \leq_{d} y$
(iii) $x \leq_{p} y$ 
Then (i) $\Longrightarrow$ (ii) $\Longrightarrow$ (iii). If $M$ is a refinement, algebraically ordered and cancellative semigroup, (iii) $\Longrightarrow$ (ii). If $M$ is algebraically ordered, cancellative and simple, then all three conditions are equivalent.

Proof. (i) $\Longrightarrow$ (ii): This is trivial, noting that we always have $0<_{s} 0$.

(ii) $\Longrightarrow$ (iii): If $x \leq_{d} y$, we have $x=x_{1}+x_{2}, y=y_{1}+y_{2}$ with $x_{1} \leq y_{1}$ and $(n+1) x_{2} \leq n y_{2}$ for some $n$. Then also $n x_{2} \leq n y_{2}$ and $(n+1) x_{2} \leq(n+1) y_{2}$, so that $x_{1} \leq_{p} y_{1}$ and $x_{2} \leq_{p} y_{2}$. Thus $x \leq_{p} y$.

Now assume (iii) and also that $M$ is a refinement, algebraically ordered, cancellative semigroup. Since $x \leq_{p} y$, we have that $n x \leq n y$ and $(n+1) x \leq(n+1) y$ for some $n$. We may find then $z$ and $w \in M$ such that $n x+z=n y$ and $(n+1) x+w=(n+1) y$. As in Proposition 3.5, cancellation implies that $(n+1) z=n w$, and thus $z<_{s} w$. Also

$$
x+n x+w=n y+y=n x+z+y,
$$

whence $x+w=y+z$. By applying refinement to this equality, we find elements $x_{i j} \in M$ such that $x=x_{11}+x_{12}, y=x_{11}+x_{21}$, while $z=x_{12}+x_{22}$ and $w=x_{21}+x_{22}$. Put $x_{1}=y_{1}=x_{11}, x_{2}=x_{12}$, and $y_{2}=x_{21}$. Since $x_{12}+x_{22}=z<_{s} w=x_{21}+x_{22}$, it follows from cancellation that $x_{2}<_{s} y_{2}$.

Finally assume that $M$ is simple, algebraically ordered, and cancellative, and assume that $x \leq_{p} y$. If $x \not \leq y$, there is by cancellation a natural number $n$ such that $n x<n y$. Now find a non-zero element $z$ such that $n x+z=n y$. Since $M$ is simple and $z$ is non-zero, there is $k$ such that $(k+1) x \leq k y$, so that $x<_{s} y$.

Proposition 5.8. Let $M$ be an algebraically ordered, cancellative semigroup. Suppose that $M$ is either simple or else that $M$ is a refinement semigroup. Then $x \otimes 1 \leq y \otimes 1$ in $M \otimes Z$ if, and only if, $x \leq_{p} y$.

Proof. By Lemma 5.2 we only need to check the 'only if' direction. Thus, assume $x \leq_{p} y$.

If $M$ is simple, then Lemma 5.7 shows that $x \leq_{s} y$, and we may apply Lemma 5.2 to conclude that $x \otimes 1 \leq y \otimes 1$.

If $M$ satisfies the Riesz decomposition property, then again by Lemma 5.7, we see that $x \leq_{d} y$, so that $x=x_{1}+x_{2}$ and $y=y_{1}+y_{2}$ with $x_{1} \leq y_{1}$ and $x_{2}<_{s} y_{2}$. Using Lemma 5.2 at the second step we obtain

$$
x \otimes 1=x_{1} \otimes 1+x_{2} \otimes 1 \leq y_{1} \otimes 1+y_{2} \otimes 1=y \otimes 1,
$$

as desired.

Proposition 5.9. Let $M$ be an algebraically ordered, cancellative semigroup. Then, the following conditions are equivalent:

(i) $M \otimes 1$ is order-cancellative;

(ii) $M \otimes 1$ is order-separative;

(iii) $M \otimes 1$ is nearly unperforated;

(iv) $x \leq_{p} y \Longrightarrow x \otimes 1 \leq y \otimes 1$ in $M \otimes 1$.

Proof. It is clear that (i) $\Longrightarrow$ (ii).

Let us show that (ii) $\Longrightarrow$ (iv): Assume that $x \leq_{p} y$. Then $n x+z=n y$ and $(n+1) x+w=(n+1) y$ for some $n$, and cancellation implies that $(n+1) z=n w$ (as in the proof of Lemma 5.7), so $z \otimes 1 \leq w \otimes 1$ by Lemma 5.2. Using this at the second step, we obtain:

$$
x \otimes 1+n y \otimes 1=(n+1) x \otimes 1+z \otimes 1 \leq(n+1) x \otimes 1+w \otimes 1=y \otimes 1+n y \otimes 1 .
$$

Since $M \otimes 1$ is assumed to be separative, we obtain $x \otimes 1 \leq y \otimes 1$. 
(iv) $\Longrightarrow$ (iii): This is clear using Lemma 5.2,

(iii) $\Longrightarrow$ (i): Suppose that $x \otimes 1+z \otimes 1 \leq y \otimes 1+z \otimes 1$. Using Lemma 5.2 and cancellation on $M$ we obtain $x \leq_{p} y$. This implies $x \otimes 1 \leq_{p} y \otimes 1$ and by (iii) we have $x \otimes 1 \leq y \otimes 1$, as desired.

Corollary 5.10. Let $M$ be an algebraically ordered, cancellative semigroup. Suppose that $M$ is either simple or else that $M$ is a refinement semigroup. Then $M \otimes 1$ is order-cancellative and nearly unperforated.

Proof. By Proposition 5.8, $x \leq_{p} y \Longleftrightarrow x \otimes 1 \leq y \otimes 1$. The result then follows from Proposition 5.9.

To close this section, we relate these results to the constructions in Section 4:

Proposition 5.11. Let $M$ be an algebraically ordered, cancellative semigroup. Suppose that $M \otimes 1$ is order-cancellative. Then $\operatorname{Gr}(M)_{\mathrm{au}}^{+} \cong \operatorname{Gr}(M \otimes 1)^{++}$.

Proof. We have to define a semigroup isomorphism $\varphi: \operatorname{Gr}(M)_{\mathrm{au}}^{+} \rightarrow \operatorname{Gr}(M \otimes 1)^{++}$.

Denote by $\iota_{M}: M \rightarrow \operatorname{Gr}(M)^{+}$the Grothendieck map for $M$, and by $\iota_{M \otimes 1}^{+}: M \otimes 1 \rightarrow$ $\operatorname{Gr}(M \otimes 1)^{++}$the corresponding map for $M \otimes 1$.

Given $x \in \operatorname{Gr}(M)_{\mathrm{au}}^{+}$, write $x=\iota(a)-\iota(b)$, for $a, b \in M$. Since there is $n \in \mathbb{N}$ such that $n x$ belongs to $\operatorname{Gr}(M)^{+}=\iota(M)$ and $M$ is cancellative, we have $n b \leq n a$ in $M$. Likewise, $(n+1) b \leq(n+1) a$ as $(n+1) x \in \operatorname{Gr}(M)^{+}$. Thus $a \leq_{p} b$. Now Proposition 5.9 implies $b \otimes 1 \leq a \otimes 1$ in $M \otimes 1$. Put $\varphi(x)=\iota_{M \otimes 1}^{+}(a \otimes 1)-\iota_{M \otimes 1}^{+}(b \otimes 1)$.

In order to verify that $\varphi$ is well defined, suppose that $x=\iota(a)-\iota(b)=\iota(c)-\iota(d)$. Then $a+d=b+c$ in $M$ and thus $a \otimes 1+d \otimes 1=b \otimes 1+c \otimes 1$ in $M \otimes 1$. It follows from this that $\iota_{M \otimes 1}^{+}(a \otimes 1)-\iota_{M \otimes 1}^{+}(b \otimes 1)=\iota_{M \otimes 1}^{+}(c \otimes 1)-\iota_{M \otimes 1}^{+}(d \otimes 1)$.

It is clear that $\varphi$ is additive. To check injectivity, retain notation from the previous paragraph, and assume $\varphi(\iota(a)-\iota(b))=\varphi(\iota(c)-\iota(d))$. Since $M \otimes 1$ is order-cancellative, this means that $(a+d) \otimes 1=(c+b) \otimes 1$. Now Lemma 5.2 combined with cancellation imply $a+d=c+b$. Thus $\iota(a)-\iota(b)=\iota(c)-\iota(d)$.

Finally, given $y=\iota_{M \otimes 1}^{+}(a \otimes 1)-\iota_{M \otimes 1}^{+}(b \otimes 1) \in \operatorname{Gr}(M \otimes 1)^{++}$with $b \otimes 1 \leq a \otimes 1$, apply Lemma 5.2 to obtain $b \leq_{p} a$ and thus $x:=\iota(a)-\iota(b)$ is an element in $\operatorname{Gr}(M)_{\mathrm{au}}^{+}$ with $\varphi(x)=y$.

Corollary 5.12. Let $A$ be a $C^{*}$-algebra containing a full projection. Suppose that $A$ has real rank zero, cancellation into ideals, and $A \otimes \mathcal{Z}$ is residually stably finite. Then $V(A \otimes \mathcal{Z}) \cong \operatorname{Gr}\left(\mathrm{V}(A) \otimes 1_{Z}\right)^{++}$.

Proof. This is an application of the proof of Corollary 4.16 and Proposition 5.11.

\section{Applications to Cu-semigroups}

In this section we apply our results to the study of the structure of algebraic $\mathrm{Cu}-$ semigroups tensored with the Cuntz semigroup of the Jiang-Su algebra $\mathcal{Z}$. As a consequence, we uncover a somewhat surprising and new connection between the axiom of almost algebraic order (O5) and almost unperforation.

We start by recalling the construction of the tensor product in the category $\mathrm{Cu}$, as well as its main features, since this will be used in the remaining of the paper.

6.1 (Tensor products in the category $\mathrm{Cu}$ and algebraic $\mathrm{Cu}$-semigroups). Given a positively ordered semigroup $S$, an auxiliary relation on $S$ (see [15]) is a binary relation $\prec$ that is weaker than the order, such that $0 \prec x$ for any $x \in S$, and satisfying

$$
x \leq y \prec z \leq t \Longrightarrow x \prec t,
$$


for any $x, y, z, t \in S$.

As immediate examples of auxiliary relations, we have the order $\leq$ in any positively ordered semigroup, and also the relation « of compact contaniment.

In [4], a category PreW is defined for positively ordered semigroups that have an auxiliary relation. This category sets up a convenient framework to manufacture tensor products, that can then be brought back to $\mathrm{Cu}$. The axioms defining this category parallel those used to define the category $\mathrm{Cu}$, and will not be repeated here as they will not be explicitely needed. We mention that $\mathrm{Cu}$ is a subcategory of PreW, and that any positively ordered semigroup $S$ can be viewed as a PreW-semigroup, by taking its order as an auxiliary relation.

The category PreW admits a tensor product construction, as follows (see [4, Section 6.2] for details): given $S$ and $T$ objects in PreW with their respective auxiliary relations, then $S \otimes_{\text {PreW }} T$ is the ordinary partially ordered semigroup tensor product, as described in 5.1, equipped with a relation $\prec$ defined as follows, for $x, y \in S \otimes T$ :

$$
x \prec y \text { if whenever } y=\sum_{i} s_{i} \otimes t_{i} \text {, there are } s_{i}^{\prime} \prec s_{i}, t_{i}^{\prime} \prec t_{i} \text { with } x \leq \sum_{i} s_{i}^{\prime} \otimes t_{i}^{\prime} .
$$

This of course applies to Cu-semigroups as well, but is not enough to give the tensor product a $\mathrm{Cu}$-semigroup structure. One needs to apply a completion functor $\gamma$ : PreW $\rightarrow$ $\mathrm{Cu}$, as in [4, Section 3.1], showing that $\mathrm{Cu}$ is in fact a reflective subcategory of PreW, and thus $\gamma(S) \cong S$ precisely when $S$ is a Cu-semigroup. Therefore, for $\mathrm{Cu}$-semigroups $S$ and $T$, one has $S \otimes_{\mathrm{Cu}} T=\gamma\left(S \otimes_{\mathrm{PreW}} T\right)$ (see [4, Theorem 6.3.3]). If we start with PreW-semigroups $S$ and $T$, then it is proved in [4, Theorem 6.3.5] that $\gamma(S \otimes$ PreW $T) \cong$ $\gamma(S) \otimes_{\mathrm{Cu}} \gamma(T)$. For a PreW-semigroup $S$ with an auxiliary relation $\prec$, we will sometimes also write $\gamma(S, \prec)$ to ensure clarity.

From the completion point of view above, a $\mathrm{Cu}$-semigroup $S$ is algebraic precisely when $S=\gamma\left(S_{c}\right)$. Hence, the tensor product of an algebraic Cu-semigroup with any other Cu-semigroup $T$ can always be thought as $S \otimes_{\mathrm{Cu}} T \cong \gamma\left(S_{c} \otimes_{\mathrm{PreW}} T\right)$. We shall be using this observation below.

As proved in [4, Theorem 5.5.8], algebraic Cu-semigroups are relevant since various structural properties of $S$ are determined by corresponding properties of $S_{c}$. For example, $S$ satisfies (O5) if, and only if, $S_{c}$ is algebraically ordered. Also, $S$ has weak cancellation if, and only if, $S_{c}$ is a cancellative semigroup. If $S_{c}$ satisfies the Riesz decomposition property, then $S$ satisfies the so called axiom of almost Riesz decomposition:

Recall that a Cu-semigroup $S$ is said to satify axiom (O6) (the axiom of almost Riesz decomposition) if, whenever $x^{\prime} \ll x \leq y+z$, there exist $y_{0} \leq x, y$ and $z_{0} \leq x, z$ such that $x^{\prime} \leq y_{0}+z_{0}$. This axiom was introduced by L. Robert in [27], where it was proved that for any $\mathrm{C}^{*}$-algebra $A$, its Cuntz semigroup $\mathrm{Cu}(A)$ satisfies (O6) (see [27, Proposition 5.1.1]).

The Cuntz semigroup $\mathrm{Cu}(A)$ for a $\mathrm{C}^{*}$-algebra $A$ of real rank zero is the typical example to bear in mind of an algebraic semigroup.

We recall three of the questions that motivate this paper:

Problem 6.2. (i) If $S$ is an algebraic Cu-semigroup, does it follow that $S \otimes_{\mathrm{Cu}} Z$ is also algebraic?

(ii) Given a Cu-semigroup $S$, characterize when $x \otimes 1 \leq y \otimes 1$ in $S \otimes_{\mathrm{Cu}} Z$.

(iii) When do axioms (O5), (O6) or the condition of weak cancellation pass from $S$ to $S \otimes_{\mathrm{Cu}} Z$ ? 
We shall mostly focus on the algebraic case, since then results in previous sections will become available. Thus in the next two lemmas we analyse the order in the tensor product $M \otimes_{\mathrm{PreW}} Z$ when $M$ is an almost divisible semigroup (see Definition 3.1).

Lemma 6.3. Let $M$ be an almost divisible positively ordered semigroup. Let $x$ be an element in $M$, and $s<t$ in $(0, \infty)$. Then, there exist $n \in \mathbb{N}, y \in M$ such that $x \otimes s \leq$ $n y \otimes 1_{Z} \leq x \otimes t$ in $M \otimes$ PreW $Z$.

Proof. If $t-s \geq 1$, then there is $n$ such that $s \leq n \cdot 1^{\prime} \leq n \cdot 1_{Z} \leq t$, and so in this case $x \otimes s \leq n x \otimes 1_{Z} \leq x \otimes t$.

Suppose that $t-s<1$. Then, we may choose $L$ such that $L>\frac{1+t}{t-s}$. Then $(L-1) t-$ $L s>1$, hence we may find $n \in \mathbb{N}$ such that $L s<n<(L-1) t$. Since $M$ is almost divisible, there exists $y \in M$ such that $(L-1) y \leq x \leq L y$. Then:

$$
x \otimes s \leq L y \otimes s=y \otimes L s \leq y \otimes n 1_{Z} \leq y \otimes(L-1) t=(L-1) y \otimes t \leq x \otimes t .
$$

Lemma 6.4. Let $M$ be an almost divisible positively ordered semigroup. Then $a \prec b$ in $M \otimes \operatorname{PreW} Z$ if and only if there exists $c \prec c$ in $M \otimes_{\mathrm{PreW}} Z$ such that $a \prec c \prec b$.

Further, an element $a \in M \otimes_{\mathrm{PreW}} Z$ satisfies $a \prec a$ if and only if there is $x \in M$ such that $a=x \otimes 1_{Z}$.

Proof. Write $b=\sum_{i} x_{i} \otimes t_{i}$. Since $a \prec b$, there exist, for each $i$, elements $x_{i}^{\prime} \leq x_{i}$ and $s_{i} \ll t_{i}$ such that $a \leq \sum_{i} x_{i}^{\prime} \otimes s_{i}$. Write $Z=\mathbb{N} \sqcup(0, \infty]$, and put $I=\left\{i \mid s_{i} \in \mathbb{N} \subset Z\right\}$, and $J=\left\{i \mid s_{i} \in(0, \infty] \subset Z\right\}$. There is nothing to prove if $J=\emptyset$, as in this case we may take $c=\sum_{i \in I} x_{i}^{\prime} \otimes s_{i}$.

Assume then $J \neq \emptyset$. For each $i \in J$, we have $s_{i}<t_{i} \leq \infty$, and we may use Lemma 6.3 to find $z_{i} \in M, n_{i} \in \mathbb{N}$ such that

$$
x_{i}^{\prime} \otimes s_{i} \leq n_{i} z_{i} \otimes 1_{Z} \leq x_{i}^{\prime} \otimes t_{i} \leq x_{i} \otimes t_{i} .
$$

Then, let $c=\sum_{i \in I} x_{i}^{\prime} \otimes s_{i}+\sum_{i \in J} n_{i} z_{i} \otimes 1_{Z}$, and clearly $a \prec c \prec b$, with $c \prec c$.

If $a \prec a$, we may apply the first part of the proof with $a=b$ and conclude that $a=x \otimes 1_{Z}$ for some $x \in M$, as desired.

In the result below we answer Problem 6.2 (i) in the affirmative, assuming further that the algebraic $\mathrm{Cu}$-semigroup $S$ is almost divisible. In this case, as shown in 4 , Lemma 7.3.6], this is equivalent to the demand that the subsemigroup $S_{c}$ of compact elements is almost divisible. We remark that this is not an unreasonable condition. Indeed, if $S$ is weakly cancellative, and satisfies (O5), (O6), then $S_{c}$ is a cancellative refinement semigroup. Thus, by Lemma 3.2, $S$ is almost divisible if and only if $S_{c}$ is weakly divisible, and the latter condition will hold if and only if no non-zero quotient of $S_{c}$ has an ideal isomorphic to $\mathbb{N}$ (by [23, Proposition 2.6]). This translates into C*algebras as follows: if $A$ is a $\mathrm{C}^{*}$-algebra of real rank zero and stable rank one, then $\mathrm{Cu}(A)$ is almost divisible if and only if $\mathrm{V}(A)$ is weakly divisible. By [26, Theorem 5.8], this is equivalent to the existence of no representation of $A$ on a Hilbert space whose image meets the compact operators non-trivially.

Theorem 6.5. Let $S$ be an algebraic, almost divisible $\mathrm{Cu}$-semigroup. Then the semigroup $S \otimes_{\mathrm{Cu}} Z$ is also algebraic with $\left(S \otimes_{\mathrm{Cu}} Z\right)_{c}=S_{c} \otimes 1_{Z}$.

Proof. Since $S$ is almost divisible as a Cu-semigroup, we have that $S_{c}$ is almost divisible as a positively ordered semigroup ([4, Lemma 7.3.6]). 
Since $S=\gamma\left(S_{c}, \leq\right)$, and $Z=\gamma(Z, \ll)$ we have, using [4, Theorem 6.3.5] (see also the comments in 6.1), that

$$
S \otimes_{\mathrm{Cu}} Z \cong \gamma\left(S_{c} \otimes_{\mathrm{PreW}} Z\right) .
$$

To ease the notation, we shall then identify $S \otimes_{\mathrm{Cu}} Z$ with $\gamma\left(S_{c} \otimes_{\mathrm{PreW}} Z\right)$, and we will also use $\alpha: S_{c} \otimes_{\mathrm{PreW}} Z \rightarrow \gamma\left(S_{c} \otimes_{\mathrm{PreW}} Z\right)$ to denote the PreW-map of the Cu-completion (see [4, Definition 3.1.7 and Proposition 3.1.6]). We will denote by $\prec$ the auxiliary relation in $S_{c} \otimes$ PreW $Z$ (see 6.1 and [4, Definition 6.2.9]).

Let $a \in S \otimes_{\mathrm{Cu}} Z$. Then, by [4, Theorem 3.1.8], there exists a sequence $\left(a_{n}\right)$ in $S_{c} \otimes \operatorname{PreW} Z$ such that $a_{n} \prec a_{n+1}$ and $a=\sup \alpha\left(a_{n}\right)$ (and, in fact, $\alpha\left(a_{n}\right) \ll \alpha\left(a_{n+1}\right)$ ). By Lemma 6.4, we can find, for each $n$, elements $c_{n} \in S_{c} \otimes$ PreW $Z$ such that $a_{n} \prec c_{n} \prec$ $c_{n} \prec a_{n+1}$. This means that $\alpha\left(a_{n}\right) \ll \alpha\left(c_{n}\right) \ll \alpha\left(a_{n+1}\right)$, with $\alpha\left(c_{n}\right)$ compact for each $n$. Thus compact elements are dense and hence $S \otimes_{\mathrm{Cu}} Z$ is algebraic.

This argument also shows that, in fact, $(S \otimes Z)_{c}=\left\{\alpha(x \otimes 1) \mid x \in S_{c}\right\}=\alpha\left(S_{c} \otimes 1_{Z}\right) \cong$ $S_{c} \otimes 1_{Z}$.

We next characterize, for a wide class of algebraic Cu-semigroups $S$, when $x \otimes 1 \leq y \otimes 1$ in $S \otimes_{\mathrm{Cu}} Z$, thus giving a partial answer to Problem 6.2 (ii). We also address part of Problem 6.2 (iii).

Theorem 6.6. Let $S$ be an algebraic, almost divisible $\mathrm{Cu}$-semigroup. Suppose that $S$ has weak cancellation and satisfies (O5), (O6). Then:

(i) $S \otimes_{\mathrm{Cu}} Z$ is nearly unperforated and weakly cancellative.

(ii) For $x, y \in S, x \otimes 1_{Z} \leq y \otimes 1_{Z}$ in $S \otimes_{\mathrm{Cu}} Z$ if and only $x^{\prime} \leq_{p} y$ for all $x^{\prime} \ll x$.

Proof. Retain the notation in the proof of Theorem 6.5,

(i). We know that $S \otimes_{\mathrm{Cu}} Z$ is algebraic. Hence, in order to check that $S \otimes_{\mathrm{Cu}} Z$ is weakly cancellative and nearly unperforated, we need to show that the subsemigroup of compact elements is cancellative and nearly unperforated and then use [4, Theorem 5.5.8, Lemma 5.6.16].

Our assumptions imply that $S_{c}$ is an algebraically ordered, cancellative, refinement semigroup. Thus, by Corollary 5.10, $S_{c} \otimes 1_{Z}$ is cancellative and nearly unperforated. Finally, we have $(S \otimes Z)_{c} \cong S_{c} \otimes 1_{Z}$ from Theorem 6.5.

(ii). Suppose first that $x^{\prime} \leq_{p} y$ whenever $x^{\prime} \ll x$. Write $x=\sup s_{n}$ where $\left(s_{n}\right)$ is a rapidly increasing sequence consisting of compact elements. For each $n$, we have $s_{n} \leq_{p} y$, and therefore $\alpha\left(s_{n} \otimes 1\right) \leq_{p} y \otimes 1$. By (i), $S \otimes_{\mathrm{Cu}} Z$ is nearly unperforated, and thus $\alpha\left(s_{n} \otimes 1\right) \leq y \otimes 1$ for each $n$. Therefore $x \otimes 1 \leq y \otimes 1$.

Conversely, suppose that $x \otimes 1 \leq y \otimes 1$ and take $x^{\prime} \in S$ with $x^{\prime} \ll x$. Find a compact element $c$ in $S$ such that $x^{\prime} \ll c \ll x$. Then we have $\alpha(c \otimes 1) \ll \alpha(c \otimes 1) \leq y \otimes 1$. Let $\left(t_{m}\right)_{m}$ be a sequence of compact elements such that $y=\sup t_{m}$. Thus $y \otimes 1=\sup \alpha\left(t_{m} \otimes 1\right)$. It follows that there is $k \in \mathbb{N}$ with $\alpha(c \otimes 1) \leq \alpha\left(t_{k} \otimes 1\right)$. Using [4, Theorem 3.1.8 (1)], we get $c \otimes 1 \leq t_{k} \otimes 1$. Now using Lemma 5.2 we have $c \leq_{p} t_{k} \leq y$, and hence $x^{\prime} \leq_{p} y$.

Remark 6.7. A priori it is not clear that, even when $S$ is algebraic, almost divisible and satisfies (O5), (O6), the semigroup $S \otimes_{\mathrm{Cu}} Z$ will satisfy (O5) (more about this in the results below). If that were the case, then near unperforation as in Theorem 6.6 (i) would be automatic by virtue of [4, Proposition 5.6.12], as $S \otimes_{\mathrm{Cu}} Z$ is always almost unperforated.

Notice also that, under the assumptions of Theorem 6.6, the map $S \rightarrow S \otimes_{\mathrm{Cu}} Z$ given by $x \mapsto x \otimes 1_{Z}$ is an order-embedding if and only if $S$ is nearly unperforated, if and 
only it is an order-isomorphism. This uses statement (i) in the said theorem, and [4, Theorem 7.3.11, Theorem 7.1.12].

Corollary 6.8. Let $A$ be a $C^{*}$-algebra with real rank zero.

(i) If $\mathrm{Cu}(A)$ is almost divisible, then $\mathrm{Cu}(A) \otimes_{\mathrm{Cu}} Z$ is an algebraic $\mathrm{Cu}$-semigroup.

(ii) Furthermore, if $A$ has stable rank one, then $\mathrm{Cu}(A) \otimes_{\mathrm{Cu}} Z$ has weak cancellation and is nearly unperforated.

Proof. This follows from Theorem 6.5.

We now turn our attention to Problem 6.2 (iii). More concretely, we look into the question of whether (O5) passes from an algebraic Cu-semigroup $S$ to $S \otimes_{\mathrm{Cu}} Z$.

Theorem 6.9. Let $S$ be an algebraic, almost divisible $\mathrm{Cu}$-semigroup. Suppose that the subsemigroup $S_{c}$ of compact elements is separative. Consider the following conditions:

(i) $S \otimes_{\mathrm{Cu}} Z$ satisfies (O5).

(ii) $S_{c}$ is almost unperforated.

(iii) $S$ is almost unperforated.

(iv) $S \cong S \otimes_{\mathrm{Cu}} Z$ as $\mathrm{Cu}$-semigroups.

Then (i) $\Longrightarrow$ (ii) $\Longrightarrow$ (iii) $\Longrightarrow$ (iv). If further $S$ satisfies (O5), then all conditions are equivalent.

Proof. (i) $\Longrightarrow$ (ii): By Theorem 6.5, $S \otimes_{\mathrm{Cu}} Z$ is algebraic, and the subsemigroup of compact elements is identified with $\left\{x \otimes 1_{Z} \mid x \in S_{c}\right\}$. Suppose that $(n+1) \leq n y$ in $S_{c}$. Then, by Lemma 5.2, we get $x \otimes 1 \leq y \otimes 1$ in $S \otimes_{\mathrm{Cu}} Z$. By assumption, $S \otimes_{\mathrm{Cu}} Z$ satisfies (O5), and hence the subsemigroup of compact elements is algebraically ordered, by 4 , Theorem 5.5.8]. There exists then $z \in S_{c}$ such that $x \otimes 1+z \otimes 1=y \otimes 1$, and then $(x+z) \otimes 1=y \otimes 1$. By Lemma 5.2 we obtain $n(x+z)=n y$ and $(n+1)(x+z)=(n+1) y$ for some $n$. Since $S_{c}$ is separative, we conclude $x+z=y$.

(ii) $\Longrightarrow$ (iii): This is immediate as $S$ is algebraic, hence the completion of $S_{c}$.

(iii) $\Longrightarrow$ (iv): Since $S$ is almost unperforated and almost divisible, [4, Theorem 7.3.11] applies to conclude $S \cong S \otimes_{\mathrm{Cu}} Z$.

If $S$ satisfies (O5), clearly (iv) $\Longrightarrow$ (i).

Recall that for a stably finite $\mathrm{C}^{*}$-algebra $A$, the subsemigroup of compact elements of $\mathrm{Cu}(A)$ can be identified with $\mathrm{V}(A)$. We now interpret Theorem 6.9 for $\mathrm{C}^{*}$-algebras. This connects the open problem of whether $\mathrm{V}(A)$ is always almost unperforated, for a $\mathrm{C}^{*}$-algebra $A$ of real rank zero, with the fact that $\mathrm{Cu}(A) \otimes_{\mathrm{Cu}} \mathrm{Cu}(\mathcal{Z})$ satisfies (O5).

Corollary 6.10. Let $A$ be a stably finite, separative, almost divisible $C^{*}$-algebra of real rank zero. Then the following conditions are equivalent:

(i) $\mathrm{Cu}(A) \otimes_{\mathrm{Cu}} \mathrm{Cu}(\mathcal{Z})$ satisfies (O5).

(ii) $\mathrm{V}(A)$ is almost unperforated.

(iii) $\mathrm{Cu}(A)$ is almost unperforated.

(iv) $\mathrm{Cu}(A) \cong \mathrm{Cu}(A) \otimes_{\mathrm{Cu}} \mathrm{Cu}(\mathcal{Z})$.

Remark 6.11. Note that Corollary 6.10 applies to a large class of stably finite $\mathrm{C}^{*}$ algebras of real rank zero. As already mentioned in the comments prior to Theorem 6.5. almost divisibility holds quite widely. Further, it is an open problem to determine whether all $\mathrm{C}^{*}$-algebras of real rank zero are separative (see [5]).

If we restrict to the simple case, we further obtain the following: 
Corollary 6.12. Let $A$ be a simple, non-type $I, C^{*}$-algebra of real rank zero and stable rank one. Then, the following conditions are equivalent:

(i) $\mathrm{Cu}(A) \otimes_{\mathrm{Cu}} \mathrm{Cu}(\mathcal{Z})$ satisfies (O5).

(ii) $\mathrm{V}(A)$ is almost unperforated.

(iii) $\mathrm{Cu}(A)$ is almost unperforated.

(iv) $\mathrm{Cu}(A) \cong \mathrm{Cu}(A) \otimes_{\mathrm{Cu}} \mathrm{Cu}(\mathcal{Z})$.

(v) $\mathrm{Cu}(A) \cong \mathrm{Cu}(A \otimes \mathcal{Z})$.

Proof. By [26, Proposition 5.3], $A$ is weakly divisible, and thus $\mathrm{Cu}(A)$ is almost divisible (by [4, Lemma 7.3.6]). Since $A$ has stable rank one, it is certainly separative, an thus the equivalence between (i) and (iv) follows from Corollary 6.10.

Assume condition (iv). Then $\mathrm{Cu}(A)$ is almost unperforated and almost divisible, and we know that $\mathrm{Cu}(A) \cong V(A)^{\times} \sqcup L(F(\mathrm{Cu}(A)))$ (see, e.g. [11, Theorem 2.6], [1, Theorem 6.3], [4, Theorem 7.6.6]). On the other hand, $\mathrm{Cu}(A \otimes \mathcal{Z}) \cong \mathrm{V}(A \otimes \mathcal{Z}) \sqcup L(F(\mathrm{Cu}(A \otimes \mathcal{Z})))$. Since $\mathcal{Z}$ has a unique trace, we see that $F(\mathrm{Cu}(A \otimes \mathcal{Z})) \cong F(\mathrm{Cu}(A))$. Since $A$ is simple and has stable rank one, $A \otimes \mathcal{Z}$ also has stable rank one ([29, Theorem 6.7]). Since we also know that $\mathrm{V}(A)$ is almost unperforated, Theorem 4.13 coupled with Proposition 4.3 imply that $\mathrm{V}(A \otimes \mathcal{Z}) \cong \mathrm{V}(A)$. Therefore $\mathrm{Cu}(A) \cong \mathrm{Cu}(A \otimes \mathcal{Z})$.

That (v) implies (iv) is clear because $\mathrm{Cu}(A \otimes \mathcal{Z})$ is always almost unperforated and almost divisible.

\section{REFERENCES}

[1] R. Antoine, J. Bosa, and F. Perera, Completions of monoids with applications to the Cuntz semigroup, Internat. J. Math. 22 (2011), no. 6, 837-861. MR 2812090 (2012d:46146)

[2] R. Antoine, J. Bosa, F. Perera, and H. Petzka, Geometric structure of dimension functions of certain continuous fields, J. Funct. Anal. 266 (2014), no. 4, pp. 2403-2423. MR 3150165

[3] R. Antoine, F. Perera, and L. Santiago, Pullbacks, $C(X)$-algebras, and their Cuntz semigroups, J. Funct. Anal. 260 (2011), no. 10, pp. 2844-2880. MR 2774057 (2011m:46110)

[4] R. Antoine, H. Thiel, and F. Perera, Tensor products and regularity properties of Cuntz semigroups, Mem. Amer. Math. Soc., to appear.

[5] P. Ara, K. R. Goodearl, K. C. O'Meara, and E. Pardo, Separative cancellation for projective modules over exchange rings, Israel J. Math. 105 (1998), pp. 105-137. MR 1639739 (99g:16006)

[6] B. Blackadar, Rational $C^{*}$-algebras and nonstable $K$-theory, Proceedings of the Seventh Great Plains Operator Theory Seminar (Lawrence, KS, 1987), Rocky Mountain J. Math. 20 (1990), no. 2, 285316. MR 1065831 (92e:46135)

[7] E. Blanchard, E. Kirchberg, Non-simple purely infinite $C^{*}$-algebras: the Hausdorff case, J. Funct. Anal. 207 (2004), 461-513. MR MR2032998 (2005b:46136)

[8] L. G. Brown, G. K. Pedersen, Non-stable K-Theory and extremally rich $C^{*}$-algebras, J. Funct. Anal. 267 (2014), no. 1, 262-298. MR 3206515

[9] N. P. Brown, A. Ciuperca, Isomorphism of Hilbert modules over stably finite $C^{*}$-algebras, J. Funct. Anal. 257 (2009), no. 1, 332-339. MR 2523343 (2010d:46077)

[10] N. P. Brown, F. Perera, and A. S. Toms, The Cuntz semigroup, the Elliott conjecture, and dimension functions on $C^{*}$-algebras, J. Reine Angew. Math. 621 (2008), 191-211. MR 2431254 (2010a:46125)

[11] N. P. Brown and A. S. Toms, Three applications of the Cuntz semigroup, Int. Math. Res. Not. IMRN (2007), no. 19, Art. ID rnm068, 14. MR 2359541 (2009a:46104)

[12] A. Ciuperca, L. Robert, and L. Santiago, The Cuntz semigroup of ideals and quotients and a generalized Kasparov stabilization theorem, J. Operator Theory 64 (2010), no. 1, 155-169. MR 2669433 (2012b:46129)

[13] A. H. Clifford, G. B. Preston, The algebraic theory of semigroups, Vol. 1, Math. Surveys, No. 7, Amer. Math. Soc., Providence, 1961. MR 0132791 (24 \# A2627)

[14] K. T. Coward, G. A. Elliott, and C. Ivanescu, The Cuntz semigroup as an invariant for C*-algebras, J. Reine Angew. Math. 623 (2008), 161-193. MR 2458043 (2010m:46101) 
[15] G. Gierz, K. H. Hofmann, K. Keimel, J. D. Lawson, M. Mislove, and D. S. Scott, Continuous lattices and domains, Encyclopedia of Mathematics and its Applications, vol. 93, Cambridge University Press, Cambridge, 2003. MR 1975381 (2004h:06001)

[16] G. A. Elliott, L. Robert, and L. Santiago, The cone of lower semicontinuous traces on a $C^{*}$-algebra, Amer. J. Math. 133 (2011), no. 4, 969-1005. MR 2823868 (2012f:46120)

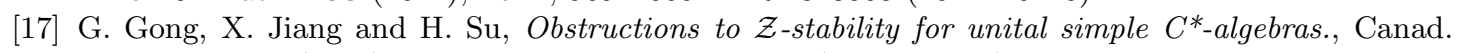
Math. Bull.,43 (2000), no. 4, 418-426. MR 1793944 (2001k:46086)

[18] X. Jiang, Nonstable K-Theory for $\mathcal{Z}$-stable $C^{*}$-algebras, arXiv:math/9707228v1 [math.OA], pages $1-18,1997$.

[19] X. Jiang and H. Su, On a simple unital projectionless $C^{*}$-algebra, Amer. J. Math. 121 (1999), no. 2, 359-413. MR 1680321 (2000a:46104)

[20] E. Kirchberg and M. Rørdam, Central sequence $C^{*}$-algebras and tensorial absorption of the Jiang-Su algebra, J. Reine Angew. Math. 695 (2014), 175-214. MR 3276157

[21] H. Matui and Y. Sato, Strict comparison and $\mathcal{Z}$-absorption of nuclear $C^{*}$-algebras, Acta Math. 209 (2012), no. 1, 179-196. MR 2979512

[22] C. Moreira Dos Santos, Decomposition of strongly separative monoids, J. Pure Applied Alg. 172 (2002), pp. 25-47. MR 1904228 (2003d:06020)

[23] E. Ortega, F. Perera, and M. Rørdam, The corona factorization property and refinement monoids, Trans. Amer. Math. Soc. 363 (2011), no. 9, 4505-4525. MR 2806681 (2012e:46130)

[24] E. Pardo, Metric completions of ordered groups and $\mathrm{K}_{0}$ of exchange rings, Trans. Amer. Math. Soc. 350 (1998), no. 3, 913-933. MR 1376552 (98e:46088)

[25] F. Perera, Extremal richness of multiplier and corona algebras of simple $C^{*}$-algebras with real rank zero, J. Operator Theory 44 (2000), 413-431. MR 1794825 (2001m:46123)

[26] F. Perera, M. Rørdam, AF-embeddings into $C^{*}$-algebras of real rank zero, J. Funct. Anal. 217 (2004), pp. 142-170. MR 2097610 (2005g:46109)

[27] L. Robert, The cone of functionals on the Cuntz semigroup, Math. Scand. 113 (2013), no. 2, 161186. MR 3145179

[28] L. Robert and L. Santiago, Classification of $C^{*}$-homomorphisms from $C_{0}(0,1]$ to a $C^{*}$-algebra, J. Funct. Anal. 258 (2010), no. 3, 869-892. MR 2558180 (2010j:46108)

[29] M. Rørdam, The stable and the real rank of $\mathcal{Z}$-absorbing $C^{*}$-algebras, Internat. J. Math. 15 (2004), no. 10, 1065-1084. MR 2106263 (2005k:46164)

[30] M. Rørdam and W. Winter, The Jiang-Su algebra revisited, J. Reine Angew. Math. 642 (2010), 129-155. MR 2658184 (2011i:46074)

[31] Y. Sato, Trace spaces of simple nuclear $C^{*}$-algebras with finite-dimensional extreme boundary, preprint (arXiv:1209.3000 [math.OA]), 2012.

[32] A. Toms, On the classification problem for nuclear $C^{*}$-algebras, Ann. of Math. (2) 167 (2008), no 3, 1029-1044. MR 2415391

[33] A. Toms and W. Winter, The Elliott conjecture for Villadsen algebras of the first type, J. Funct. Anal. 256 (2009), no. 5, 1311-1340. MR 2490221

[34] A. S. Toms, S. White, and W. Winter, $\mathcal{Z}$-stability and finite dimensional tracial boundaries, Int. Math. Res. Not. IMRN (2015), no. 10, 2702-2727. MR 3352253

[35] F. Wehrung, Restricted injectivity, transfer property and decompositions of separative positively ordered monoids, Comm. Algebra 22 (1994), no. 5, 1747-1781. MR 1264740 (94m:06017)

[36] F. Wehrung, Injective positively ordered monoids. I, II, J. Pure Appl. Algebra 83 (1992), no. 1, 43-82, 83-100. MR 1190444 (93k:06023)

[37] F. Wehrung, The universal theory of ordered equidecomposability types semigroups, Canad. J. Math. 46 (1994), no. 5, 1093-1120. MR 1295133 (95i:06025)

[38] F. Wehrung, Tensor products of structures with interpolation, Pacific J. Math. 176 (1996), no. 1, 267-285. MR 1433994 (98e:06010)

[39] W. Winter, Decomposition rank and $\mathcal{Z}$-stability, Invent. Math. 179 (2010), no 2, 229-301. MR 2570118

[40] W. Winter, Nuclear dimension and $\mathcal{Z}$-stability of pure $C^{*}$-algebras, Invent. Math. 187 (2012), no. 2, 259-342. MR 2885621

[41] W. Winter and J. Zacharias, The nuclear dimension of $C^{*}$-algebras, Adv. Math. 224 (2010), no. 2, 461-498. MR 2609012 
Ramon Antoine, Francesc Perera, Departament de Matemàtiques, Universitat Autònoma de Barcelona, 08193 Bellaterra, Barcelona, Spain

E-mail address: ramon@mat.uab.cat

E-mail address: perera@mat.uab.cat

Henning Petzka, Mathematisches Institut, Universität Münster, Einsteinstrasse 62, 48149 MÜnster, Germany

E-mail address: petzka@uni-muenster.de 